\title{
Neuron-Glia Signaling Regulates the Onset of the Antidepressant Response
}

Vicky Yao ${ }^{\# 12}$, Ammar Aly ${ }^{3}$, Salina Kalik ${ }^{3}$, Jodi Gresack ${ }^{3}$, Wei Wang ${ }^{4}$, Annie Handler ${ }^{3}$, Anne Schaefer ${ }^{5}$, Olga Troyanskaya ${ }^{26}$, Paul Greengard ${ }^{3}$, and Revathy U. Chottekalapanda ${ }^{\# \text { * }}$

1Department of Computer Science, Rice University, Houston, TX 77005, USA;

2Department of Computer Science, Princeton University, Princeton, NJ 08544, USA; Lewis-Sigler Institute for Integrative Genomics, Princeton University, Princeton, NJ 08544, USA;

${ }^{3}$ Laboratory of Molecular and Cellular Neuroscience, The Rockefeller University, New York, NY 10065, USA;

${ }^{4}$ Bioinformatics Resource Center, The Rockefeller University, New York, NY 10065, USA;

${ }^{5}$ Friedman Brain Institute, Department of Neuroscience, Icahn School of Medicine at Mount Sinai, New York, New York, USA;

${ }^{6}$ Flatiron Institute, Simons Foundation, New York, NY 10010, USA.

\# Contributed Equally

*Correspondence: rchottekal@rockefeller.edu 


\begin{abstract}
Commonly prescribed antidepressants, such as selective serotonin reuptake inhibitors (SSRls) take weeks to achieve therapeutic benefits ${ }^{1,2}$. The underlying mechanisms of why antidepressants take weeks or months to reverse depressed mood are not understood. Using a single cell sequencing approach, we analyzed gene expression changes in mice subjected to stress-induced depression and determined their temporal response to antidepressant treatment in the cerebral cortex. We discovered that both glial and neuronal cell populations elicit gene expression changes in response to stress, and that these changes are reversed upon treatment with fluoxetine (Prozac), a widely prescribed selective serotonin reuptake inhibitor (SSRI). Upon reproducing the molecular signaling events regulated by fluoxetine ${ }^{3}$ in a cortical culture system, we found that these transcriptional changes are serotonin-dependent, require reciprocal neuron-glia communication, and involve temporally-specified sequences of autoregulation and crossregulation between FGF2 and BDNF signaling pathways. Briefly, stimulation of Fgf2 synthesis and signaling directly regulates Bdnf synthesis and secretion cell-non-autonomously requiring neuron-glia interactions, which then activates neuronal BDNF-TrkB signaling to drive longer-term neuronal adaptations ${ }^{4-6}$ leading to improved mood. Our studies highlight temporal and cell type specific mechanisms promoting the onset of the antidepressant response, that we propose could offer novel avenues for mitigating delayed onset of antidepressant therapies.
\end{abstract}




\section{Introduction}

An estimated 350 million people globally suffer from depressive disorders and these disorders are a leading cause of disability and economic burden worldwide ${ }^{7}$. Selective serotonin reuptake inhibitors (SSRIs) have been used for decades as the first-line pharmacotherapy for depressive disorders due to their safety, efficacy, and tolerability, and are approved for use in both adult and pediatric patients ${ }^{1,2,8,9}$. SSRIs are effective in two-thirds of major depressive disorder (MDD) patients and take weeks before achieving mood improvement ${ }^{1,10}$. During these weeks, patients can exhibit multiple side effects, among which suicidal ideation is highly predominant ${ }^{11}$. Postmortem and neuroimaging analysis in MDD patients have identified structural and functional impairments within critical neuronal networks during depression pathophysiology ${ }^{12}$. In rodent models, antidepressants are known to raise extracellular serotonin levels within hours of treatment ${ }^{13}$, and are thought to reverse the structural and functional abnormalities by inducing long-term neuronal adaptations such as neurogenesis ${ }^{14}$, altered neuronal morphology and synaptic plasticity $5,6,15,16$. These adaptations, requiring weeks to provide mood improvement in humans and rodent models, indicate the existence of broader, conserved serotonin-dependent mechanisms during response onset which are not understood. Here, we aim to address the delayed cellular and molecular mechanism of action of antidepressants in a mouse depression model using social-isolation rearing stress paradigm and treatment with fluoxetine (Prozac), a widely prescribed SSRI.

\section{Various cortical cell types respond to stress and antidepressant treatment}

To identify the mechanisms that cause weeks-long delay during the antidepressant response, we profiled the temporal dynamics of gene expression changes in response to stress and antidepressant treatment using single-nuclei RNA sequencing (snRNAseq) from the mouse cerebral cortex, an important region in the cortico-limbic mood-circuit ${ }^{12,17}$ using the 10x genomics platform (Figure 1A). Mice were subjected to social-isolation rearing stress to induce depressive-like behavior and were treated with vehicle or fluoxetine as previously described ${ }^{3,18}$. Gene expression changes for the different groups were analyzed at intervals of early (3 days), week-long (7 days), and close to observed behavioral improvement (10 days) of treatment (Figure 1B). Stressed animals displayed anhedonia measured by sucrose preference test (Figure 1C) and exhibited heightened startle reactivity as a read-out of their emotional state when their acoustic startle response was measured (Figure 1D). To determine the gene expression changes, we first assembled an integrated dataset across all time points and treatment groups. We then identified cell populations based on the corresponding marker genes enriched in each cluster and reviewed cell type specific marker gene expression patterns in available databases (Allen Brain Atlas, GENSAT, gene expression database (GXD) and anatomical studies) ${ }^{19-25}$, and obtained 36 distinct clusters (Figure 1E). We grouped and organized these clusters into 15 neuronal (Extended Figure 1A, Extended Table 1), 13 non-neuronal (glial and 
other) Extended Figure 1B, Extended Table 2), and 5 inter-neuronal cell types (Extended Figure 1C, Extended Table 3).

To determine the molecular and cellular adaptations triggered by stress that are reversed by fluoxetine treatment, we identified differentially expressed genes that were downregulated by stress and upregulated in response to fluoxetine and genes that were upregulated by stress and downregulated by fluoxetine. We achieved this by overlaying differentially expressed genes from two comparisons: chronically stressed versus control; and chronically stressed versus chronically stressed treated with fluoxetine as depicted in the Venn diagram (Figure 1F, Extended Table 4). We found a significant overlap in the transcriptional regulation between the two comparisons in multiple cell types and identified stress-affected fluoxetinenormalized genes. We observed a steady increase in the transcriptional regulation of stress-affected fluoxetine-normalized genes along the time course of treatment. Notably, we found that the normalization of stress-induced changes occurred in cell populations that include glial, neuronal, and inter-neuronal populations, as shown by the volcano plots on day 3, 7, and 10 of treatment (Figure 1G, Extended Figure 2). These results highlight the necessity of studying the antidepressant response from the perspective of all the different cell types that work together to shape the behavioral response.

\section{Neuronal and glial responses to antidepressant treatment are temporally organized}

Next, we analyzed the dynamics of the stress-affected fluoxetine-normalized genes at the level of individual glial and neuronal cell populations by performing gene ontology (GO) analysis ${ }^{26}$. We found that both glial and neuronal populations are involved during the early antidepressant response (Figure 2A, B and Extended Table 5). We found significant transcriptional alterations in astrocytes, neurons of layers 2/3, and layer 6 at all time points on days 3, 7, and 10 of fluoxetine treatment. In addition to the above three cell types, oligodendrocyte precursor (OPC) cells also had significant differential expression on day 7 of treatment. Finally, on day 10 of treatment, there were gene expression changes observed in multiple neuronal populations, parvalbumin expressing (PV) interneurons, astrocytes and mature oligodendrocytes (Figure 2A, B and Extended Table 5). Changes in mature oligodendrocytes were observed only on day 10 of treatment, concurrent with alterations in multiple neuronal populations. These observations reveal a highly organized response to fluoxetine treatment by the glial and neuronal populations.

Distinct functions were identified for the stress-affected fluoxetine-normalized gene changes in these cell types as determined by GO analysis and pathway analysis (Figure 2B). Select genes representing celltype specific cellular functions are represented in Extended Figure 3 right panel. Astrocyte changes were observed at all three tested times of treatment. Astrocyte gene changes were attributed to cell projection, cytoskeletal organization, and axonal guidance signaling on days 3, 7 of treatment. In addition, for day 7 of treatment, pathways relating to axonal development, neurotrophin signaling, formation of dendrites, and 
OPC regulation were observed. Response from multiple neuronal populations were observed on day 10 of treatment, highlighting alterations in neuronal morphology, neurotransmission, and lipid metabolism (Figure 2B and Extended Figure 3). Neuronal remodeling and plasticity inducing properties of synaptic long-term potentiation, protein kinase A signaling, synaptogenesis signaling pathway, and cholesterol metabolism were observed. In addition, on day 10 of treatment, mature oligodendrocyte functions contributing to myelination, neuregulin signaling, ephrin receptor signaling, and axonal guidance signaling were observed.

To further characterize the cell type specific functions, we identified upstream regulators modulating stressaffected fluoxetine-regulated genes in glial and neuronal populations. We identified cluster-specific potential regulators such as TCF7L2, RxRB, VEGF, BDNF, and FGF2, molecules that have been previously shown to play a central role in the regulation of stress response in rodent models, depressivelike behavior, and antidepressant responses ${ }^{5,27-30}$ (Extended Figure 3 middle panel). These molecules have known functions in neuronal plasticity and remodeling pathways, confirming that long-term neuronal adaptations take time to occur. Collectively, these results support the possibility that early changes in the glial cells support late-stage neuronal adaptations contributing to improved behavior.

\section{Molecular markers to represent the fluoxetine response}

Next, we determined whether glial cell functions support late-stage neuronal adaptations that lead to the antidepressant response. First, we asked if the molecular players previously implicated in the antidepressant response had a role in the regulation of neuron-glia interactions. To do so, we chose temporal markers to follow the time course of the fluoxetine response. We had previously identified S100a10 (p11 protein) as an important molecule which is affected by stress and is essential for providing antidepressant response ${ }^{3,31}$. Hence we analyzed the dynamics of $5100 a 10$ regulation in response to fluoxetine treatment. Interestingly, we found that $\$ 100 a 10$ transcription is temporally regulated, stimulated only at the late phase, between 9 and 14 days of treatment (Figure 3A), closer to the timeline of observed behavioral improvement. This allowed us to use S100a10 transcription as a proxy for the late response phase and prompted us to identify factors that control S100a10 transcription to address the cause for the delayed response. p11 is known to be readily induced by growth factors ${ }^{32}$, and therefore, we applied potential growth-factors to stimulate S100a10 transcription in mouse primary mixed cortical cultures, a model system widely used to study the physiological properties of neurons (Extended Figure 4 A, B) ${ }^{33}$. Among tested factors, we observed a strong and rapid induction (within $2 \mathrm{~h}$ of stimulation) of S100a10 transcription by BDNF and FGF2 (3-fold) and a small induction by EGF (1.2 fold) (Figure 3B). We found that BDNF and FGF2 signaling modulated S100a10 transcription through the activation of BDNF and FGF2 receptors, TrkB and FGFRs respectively, via regulation of MAPK, PI3K, and JNK cascades (Extended Figure 4C, D, E, F). Previously, the significant role of both FGF2- and BDNF-signaling in the regulation of 
mood, as well as their requirement and sufficiency for providing antidepressant response has been well described $^{5,30}$. In addition, correlation with respect to reduced levels of both factors was observed in MDD patients and their downregulation in animal models of stress and depression. Thus, our findings and literature precedent led us to choose Fgf2, Bdnf, and S100a10 expression as temporal markers to represent the time course of the fluoxetine response.

\section{Recapitulating the molecular response to fluoxetine in a cortical cell culture system}

To address if the chosen molecules essential for the antidepressant response played a role in the regulation of neuron-glia interactions, we asked if we could mimic the molecular response to fluoxetine in a cortical cell culture system. We treated mouse primary mixed cortical cultures harboring both neuronal and glial cell types (Extended Figure 4A, B) with serotonin (5-HT), and analyzed the transcriptional regulation of Fgf2, Bdnf, and S100a10 expression. Considering that extracellular serotonin levels go up within 30 min of stimulation ${ }^{13}$, we argued that treating cultures directly with fluoxetine would be ineffective, as these cultures lacked the input from the dorsal raphe projection neurons, which provide the main source of serotonin ${ }^{34,35}$. As expected, we found that fluoxetine treatment of mixed cultures did not change the expression levels of these molecules (Extended Figure 4G). Strikingly, we found that chronic treatment of mixed cultures with serotonin stimulated Fgf2, Bdnf, and S100a10 transcription when acute treatment had no effects (Figure 3C, D). In addition, we observed temporal regulation for Fgf2 and Bdnftranscription, where Fgf2 stimulation occurred on day 7 and Bdnf stimulation occurred between days 7 and 14 of treatment. In parallel sister-cultures comprising predominantly neurons, and where glial cell numbers were largely reduced (Extended Figure 4A, B), serotonin treatment failed to stimulate the expression of these genes (Figure 3C, D). Thus, with respect to these measured temporal molecular markers, we have reproduced the early in vivo molecular response to fluoxetine in a cell culture system, providing evidence that a direct communication between neurons and glial cells is essential. This first approach provided the opportunity to study the properties and interactions between key cell types and molecules that regulate mood.

\section{Serotonin regulated Fgf2, Bdnf, and S100a10 expression within neuronal and glial cell types}

To locate which of the glial and neuronal cell types stimulated Fgf2, Bdnf, and S100a10 expression in response to chronic serotonin-treatment, we performed fluorescent in situ hybridization detection (FISH) using RNAscope Fluorescent multiplex assay. To detect single molecule RNA targets at the single cell level, we labeled mixed-cultures simultaneously with RNA probes each for Fgf2, Bdnf, or S100a10 mRNA, and paired them with cell type-specific RNA probes to mark neurons and glial subtypes. We found that chronic serotonin treatment stimulated Fgf2 mRNA in astrocytes on day 7 (Figure 3E, F); Bdnf mRNA in neurons between day 10 to day 14 (Figure 3G, H); and S100a10 mRNA in neurons between day 10 to 
day 14 of serotonin treatment. Notably, Bdnf expressing neurons were distinct from those that were expressing S100a10 (Figure 3I, J). The observed effects are strictly temporal and cell type specific. For example, chronic serotonin treatment neither stimulated Fgf2 mRNA in neurons, oligodendrocytes, nor microglia (Extended Figure 5A); nor Bdnf mRNA in astrocytes or microglia (Extended Figure 5B); nor S100a10 mRNA in oligodendrocytes or microglia. Low Bdnf mRNA expression was observed in oligodendrocytes (Figure 3G, H), and low S100a10 mRNA expression was observed in astrocytes (Extended Figure 5C). Additionally, using this system, we also reproduced the temporal dynamics of Fgf2, S100a10 and Bdnf transcription in response to serotonin within neuronal and glial cell types (Extended Figure 5D, E, F). Our results demonstrate that the stimulation of key mood-regulating molecules are temporally regulated within an intricate neuron-glia multicellular circuit.

\section{Neuron-glia secreted factor confers downstream serotonin-dependent function in neurons}

Next, we determined if the serotonin-dependent neuron-glia signaling circuit requires physical cell-cell contact or is based on the secretion of neuron-glia factors. To do so, we tested if we could reproduce the serotonin-dependent function by swapping conditioned media from mixed cultures to neuronal cultures during days 5, 7, 10, and 14 of serotonin treatment, and analyzed for S100a10 transcription (a late-stage temporal marker) (Figure 4A, B). Remarkably, we observed a strictly time-dependent induction of S100a10 in the neuronal cultures that received mixed conditioned media from days 10 and 14 and not from days 5 and 7 of serotonin treatment. We failed to observe induction of $\$ 100 a 10$ when neuronal cultures received vehicle-treated mixed media. These results indicate that serotonin-dependent neuronglia derived factors secreted from the mixed cultures are temporally regulated and are required and sufficient to stimulate S100a10 expression in neuronal cultures.

Our next goal was to identify such neuron-glia derived secretory factors. Best inducible S100a10 expression was achieved when media was transferred from mixed to neuronal cultures on day 10 of serotonin treatment (Figure 4A, B). Also, at that same time point, peak stimulation of Bdnf transcription was observed (Figure $\mathbf{3 C}$ ). Therefore, we considered that BDNF could be a potential secreted-factor released from the serotonin-treated mixed cultures that could stimulate S100a10 expression in neuronal cultures. Hence, we tested this hypothesis using two strategies. We either depleted secreted BDNF from the serotonin-treated mixed-media before transfer using TrkB-Fc to chelate BDNF ${ }^{36}$ or we blocked the TrkB receptor using an inhibitor for Trk receptor autophosphorylation (K252a) ${ }^{37,38}$ that blocks BDNF/TrkB signaling. Both strategies inhibited the upregulation of $S 100 a 10$ in neuronal cultures (Figure 4C, D). These results demonstrated that Bdnf synthesis and its action on TrkB are both critical steps during serotonin treatment. Additionally, we demonstrated that Bdnf synthesis requires neuron-glia interactions and further activation of BDNF-TrkB signaling in neurons is sufficient to upregulate S100a10 transcription. 


\section{Neuron-glia dependent cross talk between FGF2 and BDNF signaling}

To gain further insight into the neuron-glia dependent regulation of Bdnf synthesis, we analyzed FGF2and BDNF-dependent regulation of S100a10 transcription in mixed and neuronal cultures. We found that FGF2 induced S100a10 transcription only in mixed cultures, whereas BDNF induced S100a10 transcription in both mixed and neuronal cultures (Figure 5A upper panel, Extended figure 6A, B). These results indicated that upstream FGF2-signaling is likely connected to Bdnf synthesis requiring neuron-glia signaling.

Next, we analyzed the reciprocal interactions between FGF2 and BDNF-signaling, and observed a strong positive autofeedback regulation. FGF2 application stimulated Fgf2 mRNA, and BDNF application stimulated Bdnf mRNA in both mixed and neuronal cultures (Figure 5A middle and lower panel, Extended figure 6C, D, E, F). Intriguingly, we observed a strong reciprocal cross-regulation, where FGF2 stimulated Bdnf mRNA only in mixed cultures and not in neuronal cultures. These results demonstrated that FGF2-dependent stimulation of S100a10 expression occurs via activation of BDNF-TrkB signaling, requiring neuron-glia interactions. Hence, we inhibited TrkB receptor activation and proved that FGF2dependent stimulation of S100a10 transcription in mixed cultures occurs via activation of TrkB signaling (Figure 5B). As expected, FGF2-stimulation of S100a10 was downregulated upon inhibition of FGFR1 receptor (predominantly bound to FGF2) and BDNF-stimulation of S100a10 was downregulated upon inhibition of TrkB receptor activation in both mixed and neuronal cultures (Figure 5B,C). These results show evidence that FGF2 stimulates $\$ 100 a 10$ expression only via regulation of $B d n f$ synthesis in a neuronglia dependent manner, and upon subsequent activation of BDNF-TrkB signaling in neurons (Figure 5C).

\section{Discussion}

Patients suffering from MDD take weeks to reverse depressed mood in response to the widely prescribed SSRI class of antidepressants. Interestingly, studies in mouse models indicate a similar timeline to achieve improved mood, suggesting that the molecular and cellular mechanisms underlying delayed response to antidepressants are likely conserved in humans and mice. Our studies provide key insights into the temporal and cell-type specific mechanisms causing the weeks-long delay in producing an antidepressant response. Here we establish that an intricate neuron-glia functional network is recruited by chronic fluoxetine/chronic serotonin treatment to initiate the response. This network involves sequential activation of FGF2-signaling in astrocytes, which directly modulates Bdnf synthesis in a neuron-glia dependent manner, and subsequent activation of BDNF-TrkB signaling facilitates neuronal adaptations (via changes in gene expression like S100a10), which in vivo eventually may lead to improved mood (Figure 5D). We 
have thus identified a critical missing step in our understanding of the delayed mechanism of action of antidepressants.

We highlight a timely and ordered neuron-glia functional organization regulating the fluoxetine response. Evidence comes from the early and persistent gene expression changes observed in astrocytes, changes in OPCs on day 7 , followed by neuronal adaptations occurring in multiple neuronal populations and mature oligodendrocytes on day 10 of treatment. Neuron-glia interactions regulate the development and maintenance of the nervous system ${ }^{39-41}$, and the contributions of glial (astrocytes, oligodendrocytes) and specific neuronal populations in the regulation of mood has been previously reported ${ }^{12,16,42-44}$. Ablation of astrocytes is shown to affect depressive-like behavior ${ }^{45}$ and corticolimbic patterns of oligodendrocyte changes are known to occur during $\mathrm{MDD}^{46,47}$. Reproducing the molecular response to antidepressant treatment in a cortical cell-culture system made it feasible to characterize the reciprocal neuron-glia communication and address the precise temporal regulation by FGF2 and BDNF signaling. It is intriguing that extracellular serotonin takes 7 days to trigger Fgf2 synthesis in astrocytes in the culture system, although we were able to document overall transcriptional changes in astrocytes and layer 2/3 and 6 neuronal populations on day 3 in vivo. It is likely that this delay could be due to the serotonin-dependent activation of yet another cell type, whose function leads to the regulation of Fgf2 synthesis in astrocytes; or simply because the surface regulation of serotonin receptors in neurons or glia require new transcription, translation, or signal transduction events.

Our results confirm that FGF2 signaling and BDNF signaling play a fundamental role in mediating the antidepressant responses ${ }^{5,30}$, but we also establish here that glial cells work in concert with neurons to facilitate response onset, subsequently promoting later long-term neuronal adaptations. Conditionedmedia swap experiments indicate that BDNF is the neuron-glia derived secreted factor that is sufficient to induce S100a10 expression (marker for the late phase of the response when behavior could be measured) in neurons. Hence we could argue that it is possible to bypass the neuron-glia interactions and reduce the delay by providing BDNF. Administering BDNF into brains of rodent depression models is known to produce a robust antidepressant response ${ }^{5}$. However, the source of Bdnf synthesis is embedded within an intricate neuron-glia interaction. As to the location of the cell type that harbors Bdnf synthesis after serotonin treatment, our in situ experiments show stimulation of Bdnf in neurons. Also, application of BDNF to neuronal cultures stimulates massive Bdnf synthesis (Figure 4D). It is likely that we missed the exact cell type that synthesizes Bdnfor that there are other factor(s) secreted from astrocytes together with FGF2 in response to serotonin, which then facilitates Bdnf synthesis in neurons. With prior evidence for Bdnf synthesis in other glial cells, such as astrocytes, oligodendrocytes, or microglia ${ }^{48-50}$, it is possible that a small amount of Bdnf is synthesized in glial cells, which then stimulates bulk Bdnf synthesis predominantly in neurons (Figure 3G, H). 
Modulation of Bdnf synthesis by FGF2 signaling is a critical molecular step, that explains an avenue by which reciprocal neuron-glia interactions are maintained. A prior study documents such a regulation, where FGF2 signaling stimulates the expression of Bdnf and of its receptor TrkB, post-axotomy of the optic nerve, in order to promote survival of frog retinal ganglion cells ${ }^{51}$. In addition, FGF2 signaling is known to promote astrocyte proliferation ${ }^{52}$, control timely differentiation of oligodendrocytes ${ }^{53}$, and interfere with FGF2 signaling in OPCs to induce depressive-like behavior ${ }^{54}$. These observations, together with the positive autofeedback regulation and cross-regulation between FGF2 and BDNF signaling pathways, indicate that maintenance of multicellular interactions are essential for normal brain function.

Our study provides a greater understanding of the pathophysiology of stress-induced depression and mechanism of action of antidepressants. We establish that neurotrophic pathways of FGF2 and BDNF signaling tightly controls the functioning of an intricate neuron-glia cell circuit to initiate the antidepressant response. We speculate that sustained antidepressant response is possible only upon resetting the disrupted neuron-glia cell circuit by the consequent activation of neurotrophic signaling pathways. Supporting this idea are the observed deficits of reduction in gray matter volume and glial cell density in MDD patients and in animal models of depression 42,55 ; reduced levels of FGF2 and BDNF during stress, depressive-like disorders, and their reversal during antidepressant treatment ${ }^{5}$; and the fact that tissue homeostasis is controlled by growth-factor signaling ${ }^{56}$. Importantly, clinical data indicates that responders to treatment show signs of improvement at day 10 of treatment ${ }^{11,57-59}$, an early time window when we document adaptations in multiple neuronal populations. We speculate that susceptibility to depressive disorders and treatment resistance could be caused due to dysregulated neuron-glia properties during this early stage. Future investigations to dissect the intricate steps of neuron-glia reciprocal signaling in vivo within the cortico-limbic mood circuit could allow for finetuning of the emerging measures to treat mood disorders by modulating neural-circuit dysfunction ${ }^{60}$. Our in vitro model system provides a feasible avenue to test for novel antidepressant medications. Further characterizing the critical role for neuron-glia reciprocal interactions during stress and antidepressant response will not only expand our understanding of mechanisms controlling brain homeostasis and repair, but also reveals key features underlying the regulation of complex behaviors.

\section{Methods}

Animals. Mice were single or pair-housed with a $12 \mathrm{~h}$ light/dark cycle (lights on from 0700 to $1900 \mathrm{~h}$ ) at constant temperature $\left(23^{\circ} \mathrm{C}\right)$ with ad libitum access to food and water. All animal protocols were approved by the Institutional Animal Care and Use Committee (IACUC) at The Rockefeller University.

Social isolation rearing stress and drug treatment in mice. C57BL/6 background FVB-Tg(S100a10EGFP/Rpl10a) ES691Htz/J mice were subjected to a social-isolation stress paradigm by single housing from P21 until P70 (single housed throughout behavior testing), and the control mice were pair-housed. Both the control and stress cohorts were treated with $0.167 \mathrm{mg} / \mathrm{ml}$ fluoxetine hydrochloride (Sigma) in 1\% saccharine solution in drinking water as described ${ }^{18}$ for 3,7 , or 10 days. For the PFC fluoxetine experiments, BALB/cJ mice were used as described ${ }^{3}$. 


\section{Behavior Analysis}

Sucrose preference test (SPT), a reward based test to measure anhedonia. Mice were presented with two dual bearing sipper tubes in their home cage. One contains plain drinking water and the second contains $2 \%$ sucrose solution. Water and sucrose solution intake was measured at $20 \mathrm{~h}$ with the positions of the bottles switched during the test to preclude side bias. Sucrose preference is calculated as a percentage of the volume of sucrose intake over the total volume of fluid intake averaged over the testing period. Pair-housed mice were temporarily single housed during the course of SPT.

Acoustic startle response to measure emotional behavior. The acoustic startle reflex refers to the contraction of the skeletal musculature in response to a high intensity acoustic stimulus. Although the acoustic startle response is a readout for reflexive behavior, this behavior is modulated by emotional state and is an indicator of arousal response. Startle reflex is a physiological measure of emotional regulation. Mice were placed in an animal holder mounted on a startle measuring platform. After an acclimation period, the amplitude of the startle response to acoustic pulse stimuli ranging from 80-120 dB was measured and recorded to determine startle reactivity.

Single nuclei purification from the mouse cortex and single cell sequencing using 10X genomics. Mouse cerebral cortex was dissected from 12-week old mice in dissection buffer containing $1 \mathrm{x} \mathrm{HBSS}, 2.5 \mathrm{mM} \mathrm{Hepes}-\mathrm{KOH}$ $\mathrm{pH} 7.4,35 \mathrm{mM}$ Glucose, $4 \mathrm{mM} \mathrm{NaHCO}$. A total of $n=24$ samples per timepoint comprising six animals per treatment group of Control, ControlFIx, Stress, and StressFlx was collected. Purification of nuclei was achieved as previously described ${ }^{61,62}$, with modifications. Each cortex was homogenized with a glass Dounce homogenizer (Kimble Chase; 1984-10002) in a lysis buffer containing $20 \mathrm{mM} \mathrm{HEPES} \mathrm{KOH} \mathrm{pH} \mathrm{7.4,} 150 \mathrm{mM} \mathrm{KCl}, 5 \mathrm{mM} \mathrm{MgCl} 2$ and the nuclei was pelleted by centrifugation at $1,200 \mathrm{xg}, 10 \mathrm{~min}, 4^{\circ} \mathrm{C}$. Nuclei was further purified in a $29 \%$ iodixanol cushion containing $0.25 \mathrm{M}$ sucrose, $25 \mathrm{mM} \mathrm{KCl}, 5 \mathrm{mM} \mathrm{MgCl}, 20 \mathrm{mM}$ tricine $\mathrm{pH} 7.8$ buffer, and myelin floats were removed during each spin. The resulting nuclear pellet was resuspended in a resuspension buffer $(0.25 \mathrm{M}$ sucrose, $25 \mathrm{mM} \mathrm{KCl}, 5 \mathrm{mM}$ $\mathrm{MgCl}_{2}, 20 \mathrm{mM}$ tricine $\mathrm{pH}$ 7.8, EDTA-free protease inhibitor cocktail (Roche, 11836170001)). 6 nuclei samples from each treatment condition was pooled. Nuclei was counted using Countess Cell counter (Thermo Fisher Scientific). 5000 nuclei per sample was processed using 10X genomics using the Chromium Single Cell 3' Library \& Gel Bead Kit v2 (10X Genomics) as per manufacturers protocol. Samples and reagents were prepared and loaded into the chip and droplets were generated using chromium controller for droplet generation. Reverse transcription was conducted in the droplets. cDNA was recovered through de-emulsification and bead purification. Pre-amplified cDNA was further subjected to library preparation and libraries were sequenced on an Illumina Next Seq 500 sequencer high output flow cell as $26 \times 57 \times 8$. Four libraries from each timepoint were pooled together and sequenced on two Next Seq lanes (totally 8 lanes).

Single cell Data processing and integration. Seurat's (v3.1.1 $)^{63}$ was used for dimensionality reduction, clustering, visualization, and differential expression analysis for the snRNAseq data. Individual Seurat count matrices was generated from sample output files from CellRanger and subsequently combined into a merged dataset. The percentage of mitochondrial genes were calculated per sample, and per Seurat's QC recommendations, cells with more than 5\% mitochondrial reads, fewer than 500 genes, or more than 5000 identified genes were filtered out. SCTransform was used to normalize and scale the data, including percentage of mitochondrial reads to regress out during the normalization process, and subsequently integrated into a single dataset.

Single cell cluster identification and annotation. Seurat's shared nearest neighbor graph-based Louvain modularity optimization approach was used to cluster the cells. Clustree (v0.4.0) ${ }^{64}$ was used to choose the appropriate resolution for clustering (0.4). Marker genes consistent for each cluster (across different treatment conditions) were identified via differential expression analysis between cells in each cluster versus all other cells (Wilcoxon rank sum test). Clusters were then assigned based on the identified marker genes. More specifically, cortical neurons were assigned layer-specific identity based on the expression of layer-specific marker genes (layer $2 / 3$, layer $4 / 5$ a, layer 5 , layer 6 and Claustrum). Multiple layer subtypes were found (they are likely the same layers from different sub-regions of the cortex), and were labeled with additional numbers (e.g., layer 2/3-1 and layer 2/32). Two small populations of neurons were ungrouped and hence called N1 and N2. There were 6 inter-neuronal (IN) populations. A small population of vascular smooth muscle cells (VSMCs), two microglial, an endothelial, a pericyte, and 4 astrocyte populations were identified. Four oligodendrocyte clusters were identified: Oligodendrocyte precursor cells (OPCs); newly-formed oligodendrocytes (NFOL); and two populations of mature oligodendrocytes.

Identification of fluoxetine-normalized genes and processes. For each cluster and time point $(3,7$, and 10 days of chronic fluoxetine treatment), we performed differential gene expression analyses (Wilcoxon rank sum test using Seurat) comparing the following conditions: stress versus control, fluoxetine-treated stress versus stress. Specifically, we aimed to identify genes that were affected by stress, then normalized by fluoxetine. To this end, we identified genes that were significantly differentially expressed in both conditions (FDR $<0.1$ ), and changing in opposite 
directions (i.e., $\log \mathrm{FC}>0$ in stress versus control and $\log \mathrm{FC}<0$ in fluoxetine-treated stress versus stress, or vice versa). We then performed Gene Ontology enrichment analysis (for terms in the "Biological Process" aspect of the Gene Ontology) on any cluster and time point that had more than 10 such fluoxetine-normalized genes using goana ${ }^{26}$ from the limma package (v3.38.3).

Mouse E18 Primary Embryonic Cortical Cultures. Pregnant C57BI/6 mice were purchased from Charles River. E18 Embryos were euthanized, and their cortices isolated. Cortices were digested into a single cell suspension using $0.15 \%$ Trypsin-EDTA (Gibco, cat \# 25200056), followed by mechanical trituration. Cells were then plated in Poly-DLysine coated 12-well plates, flasks (Corning, cat \# 354470, 356537) at 2000 cells $/ \mathrm{mm}$ cell density. Cells were initialing plated in DMEM supplemented with 10\% FBS and 1\% Penicillin-Streptomycin (Gibco, Cat \# 11960069, $10082147,15070063)$. For neuronal cultures, plating media was replaced with Neurobasal Media supplemented with B27 and N2 (Thermo Fisher Scientific, Cat \# 21103-049, 17504044, A1370701) 3h after initial cell plating. For mixed cultures, half the plating media was replaced as described above, $18 \mathrm{~h}$ after initial cell plating. For maintenance, half the media was then replaced with Neurobasal media supplemented with B27 and N2 every 3 days. For mixed glia cultures, half the plating media was replaced with plating media 18 hours following initial plating. Half the media was regularly replaced with $10 \%$ FBS supplemented DMEM every 3 days. RNA purification from the mixed and the neuronal cortical cultures, that were treated with growth factors FGF2/BDNF and kinase inhibitors; quantitative reverse-transcription and PCR (qRT-PCR), probes for Bdnf, fgf2, and S100a10, and qRT-PCR analysis were all done as described before $^{3}$. For chronic serotonin stimulation experiments, 30 uM of 5-hydroxytryptamine hydrochloride (5HT) (Millipore Sigma) was applied into DIV5 cortical neuronal and cortical mixed cultures daily, and continued for 14 days. For acute stimulation, serotonin (5-HT) was added only on the day of collection. Cells were harvested $8 \mathrm{~h}$ after stimulation at each timepoint.

RNAscope in Situ Hybridization using RNAscope Multiplex Fluorescent Assay. Mouse primary embryonic mixed cortical cultures were grown in coverslips (NeuVitro, cat \#. GG-18-15-PDL) at $2000 \mathrm{cells} / \mathrm{mm}$ cell density. The mixed cultures were treated Fgf2, Bdnf, and S100a10 RNA probes and paired them with cell-type specific RNA probes for neurons and glial subtypes to detect single molecule RNA targets at the single cell level. In situ hybridization was done using RNAscope Multiplex Fluorescent Reagent Kit v2 (ACD Bio, Cat \#. 323100) as per manufacturers protocol. Briefly, cells were fixed with 4\% PFA in PBS, dehydrated using sequential treatment with $50 \%, 75 \%$, and $100 \%$ ethanol for $5 \mathrm{~min}$, and the coverslips were adhered to superfrost glass slides. The cells were rehydrated using sequential treatment with $100 \%, 75 \%$, and $50 \%$ ethanol for 5 min and permeabilized with $0.1 \%$ Tween in PBS. Endogenous peroxidases were blocked using hydrogen peroxide treatment and extracellular proteases were blocked using protease III treatment. RNAscope probes from ACD Bio were hybridized at $40^{\circ} \mathrm{C}$. The probes used were as follows: Mm-Fgf2, 316851; Mm-Bdnf-C4, 424821-C4; Mm-S100a10-C2, 410901; Mm-Rbfox3C2, 313311-C2; Mm-Gfap-C3, 313211-C3; Mm-Csfr1-C2 319141-C2; Mm-Olig2-C3, 447091-C3. Tyramide amplification was performed with the Opal 520, 570, 620, and 690 reagent kits (FP1487001KT, FP1488001KT, FP1495001KT, and FP1497001KT), (Akoya Biosciences) for $1 \mathrm{~h}$ at $40^{\circ} \mathrm{C}$. Coverslips were mounted using ProLong Gold mount and imaged using a Zeiss LSM780 AxioObserver confocal microscope equipped with a Plan-Apochromat 100x/1.4 Oil DIC M27 lens.

Localization of Bdnf, Fgf2, and S100a10 RNA in various cell types of neurons, astrocytes, oligodendrocytes and microglia were analyzed. RNA-FISH signal was quantified using the spot counting algorithm as described ${ }^{65}$. Briefly, using ImageJ's particle analysis feature with a minimum spot size of $0.77 \mu \mathrm{m}$, spots were deduplicated along the Zaxis before further processing. Based on the nearest cell identity spot within a radius of $10 \mu \mathrm{m}$, each Bdnf, Fgf2, and S100a10 RNA signal spot was assigned to one of the four cell types (astrocytes, microglia, neurons, or oligodendrocytes). Signal spots within $10 \mu \mathrm{m}$ of multiple cell identity spots were excluded unless its nearest neighbor was at least $5 \mu \mathrm{m}$ closer than its next nearest neighbor. $40 \%$ of all detected spots were assigned to one of the four cell types with the described criteria. Eight representative images for the localization of each gene per time point was quantitated.

Expression levels of Fgf2, S100a10, and Bdnf RNA in response to serotonin treatment were quantitated as follows. RNA expression spots were counted using ImageJ particle analysis feature and were assigned to the cell types as described as above. We had samples from 4 time points for Bdnf (days 5, 7, 10, 14 of 5-HT treatment), and 2 points each for Fgf2 and S100a10 (days 10 and 14 of 5-HT treatment) respectively. For each time point, the number of RNA spots of Bdnf, Fgf2, and S100a10 RNA in each image was counted and 8 images per cell type specific marker set was analyzed. Set 1 labeled by Csfr1 (microglia) and Gfap (astrocytes) and set 2 labeled by NeuN (neurons) and Olig2 (oligodendrocytes) and the spots are assigned to a cell type. Average RNA/cell type was then computed from the data. Hence a total of 16 data points per timepoint for each of the Bdnf, Fgf2, and S100a10 RNA datasets were analyzed. 
Quantification and Statistical Analysis. Graphs and statistical analysis was done using GraphPad Prism 9. Statistical details is included in the figure legend for each experiment. Briefly, for two group comparisons, we used two-tailed unpaired student's t-test. For multiple group comparisons, we used one-way or two-way ANOVAs and corrections were applied using the appropriate post hoc test. In all experiments, $p$ value $<0.05$ was considered significant. Bar graphs show mean values and the error bars are standard error of the mean $( \pm$ SEM). The number of animals for each experiment and biological replicates for cell culture experiments are denoted by $n$. For behavior experiments, all studies were carried out and analyzed with the experimenter blinded to the treatment group.

\section{Acknowledgements:}

We thank Drs. Marc Flajolet, Priya Rajasethupathy, Dipon D.Ghosh, Jean-Pierre Roussarie, and Ms. Debra Poulter for critical comments on the manuscript. We thank Elisabeth Griggs for assistance with graphics. This research was supported by The JPB Foundation (to P.G), NARSAD Young Investigator Grant from the Brain \& Behavior Research Foundation (to R.U.C), NIH R01 GM071966 (to O.G.T), V.Y. was supported in part by US NIH grant T32 HG003284 (to O.G.T). O.G.T. is a senior fellow of the Genetic Networks program of the Canadian Institute for Advanced Research (CIFAR).

\section{Author contributions:}

R.U.C., V.Y., A.S., O.G.T., and P.G. designed and planned the experiments. R.U.C., V.Y., and O.G.T. planned the single cell experiments. V.Y., and W.W. performed the single-cell data-analysis and interpretation. R.U.C., J.G., A.A., A.H. conducted drug treatments and mouse behavioral testing. A.A., and S.K. genotyped the mice. R.U.C., S.K., and A.A. planned and performed cell culture, serotonin treatment, inhibitor treatments, qRT-PCR and analysis. R.U.C., and A.H. performed western blots and analysis. R.U.C., and A.A. planned and performed RNAscope experiments. All authors contributed to the writing of the manuscript.

\section{Competing Financial Interests:}

The authors declare no competing interests.

\section{References}

1. Rush AJ, Trivedi MH, Wisniewski SR, Nierenberg AA, Stewart JW, Warden D et al. Acute and longer-term outcomes in depressed outpatients requiring one or several treatment steps: a STAR*D report. Am J Psychiatry 2006; 163(11): 1905-1917.

2. Uher R, Mors O, Rietschel M, Rajewska-Rager A, Petrovic A, Zobel A et al. Early and delayed onset of response to antidepressants in individual trajectories of change during treatment of major depression: a secondary analysis of data from the Genome-Based Therapeutic Drugs for Depression (GENDEP) study. $J$ Clin Psychiatry 2011; 72(11): 1478-1484.

3. Chottekalapanda RU, Kalik S, Gresack J, Ayala A, Gao M, Wang W et al. AP-1 controls the p11-dependent antidepressant response. Mol Psychiatry 2020; 25(7): 1364-1381.

4. $\quad$ Castren E, Voikar V, Rantamaki T. Role of neurotrophic factors in depression. Curr Opin Pharmacol 2007; 7(1): 18-21.

5. Duman RS, Monteggia LM. A neurotrophic model for stress-related mood disorders. Biol Psychiatry 2006; 59(12): 1116-1127.

6. Krishnan V, Nestler EJ. The molecular neurobiology of depression. Nature 2008; 455(7215): 894-902.

7. THE GLOBAL BURDEN OF DISEASE 2004 UPDATE Introduction. Global Burden of Disease: 2004 Update 2008.

8. Papakostas GI. The efficacy, tolerability, and safety of contemporary antidepressants. J Clin Psychiatry 2010; 71 Suppl E1: e03.

9. Zhou X, Teng T, Zhang Y, Del Giovane C, Furukawa TA, Weisz JR et al. Comparative efficacy and acceptability of antidepressants, psychotherapies, and their combination for acute treatment of children and 
adolescents with depressive disorder: a systematic review and network meta-analysis. Lancet Psychiatry 2020; 7(7): 581-601.

10. Machado-Vieira R, Baumann J, Wheeler-Castillo C, Latov D, Henter ID, Salvadore G et al. The Timing of Antidepressant Effects: A Comparison of Diverse Pharmacological and Somatic Treatments. Pharmaceuticals (Basel) 2010; 3(1): 19-41.

11. Jick H, Kaye JA, Jick SS. Antidepressants and the risk of suicidal behaviors. JAMA 2004; 292(3): 338-343.

12. Price JL, Drevets WC. Neural circuits underlying the pathophysiology of mood disorders. Trends Cogn Sci 2012; 16(1): 61-71.

13. Invernizzi R, Belli S, Samanin R. Citalopram's ability to increase the extracellular concentrations of serotonin in the dorsal raphe prevents the drug's effect in the frontal cortex. Brain Res 1992; 584(1-2): 322-324.

14. Malberg JE, Hen R, Madsen TM. Adult Neurogenesis and Antidepressant Treatment: The Surprise Finding by Ron Duman and the Field 20 Years Later. Biol Psychiatry 2021.

15. Kavalali ET, Monteggia LM. Targeting Homeostatic Synaptic Plasticity for Treatment of Mood Disorders. Neuron 2020; 106(5): 715-726.

16. Pittenger C, Duman RS. Stress, depression, and neuroplasticity: a convergence of mechanisms. Neuropsychopharmacology 2008; 33(1): 88-109.

17. Kovner R, Oler JA, Kalin NH. Cortico-Limbic Interactions Mediate Adaptive and Maladaptive Responses Relevant to Psychopathology. Am J Psychiatry 2019; 176(12): 987-999.

18. Sargin D, Chottekalapanda RU, Perit KE, Yao V, Chu D, Sparks DW et al. Mapping the physiological and molecular markers of stress and SSRI antidepressant treatment in S100a10 corticostriatal neurons. Mol Psychiatry 2020; 25(5): 1112-1129.

19. Cadwell CR, Bhaduri A, Mostajo-Radji MA, Keefe MG, Nowakowski TJ. Development and Arealization of the Cerebral Cortex. Neuron 2019; 103(6): 980-1004.

20. Geffers L, Herrmann B, Eichele G. Web-based digital gene expression atlases for the mouse. Mamm Genome 2012; 23(9-10): 525-538.

21. Heintz N. Gene expression nervous system atlas (GENSAT). Nat Neurosci 2004; 7(5): 483.

22. Koester SE, Insel TR. Mouse maps of gene expression in the brain. Genome Biol 2007; 8(5): 212.

23. Tasic B, Menon V, Nguyen TN, Kim TK, Jarsky T, Yao Z et al. Adult mouse cortical cell taxonomy revealed by single cell transcriptomics. Nat Neurosci 2016; 19(2): 335-346.

24. Zeisel A, Munoz-Manchado AB, Codeluppi S, Lonnerberg P, La Manno G, Jureus A et al. Brain structure. Cell types in the mouse cortex and hippocampus revealed by single-cell RNA-seq. Science 2015; 347(6226): 1138-1142.

25. Zeng H, Shen EH, Hohmann JG, Oh SW, Bernard A, Royall JJ et al. Large-scale cellular-resolution gene profiling in human neocortex reveals species-specific molecular signatures. Cell 2012; 149(2): 483-496.

26. Young MD, Wakefield MJ, Smyth GK, Oshlack A. Gene ontology analysis for RNA-seq: accounting for selection bias. Genome Biol 2010; 11(2): R14.

27. Amare AT, Schubert KO, Klingler-Hoffmann M, Cohen-Woods S, Baune BT. The genetic overlap between mood disorders and cardiometabolic diseases: a systematic review of genome wide and candidate gene studies. Trans/ Psychiatry 2017; 7(1): e1007.

28. Deyama S, Bang E, Kato T, Li XY, Duman RS. Neurotrophic and Antidepressant Actions of Brain-Derived Neurotrophic Factor Require Vascular Endothelial Growth Factor. Biol Psychiatry 2019; 86(2): 143-152.

29. Krzyzosiak A, Szyszka-Niagolov M, Wietrzych M, Gobaille S, Muramatsu S, Krezel W. Retinoid x receptor gamma control of affective behaviors involves dopaminergic signaling in mice. Neuron 2010; 66(6): 908-920. 
30. Turner CA, Watson SJ, Akil H. The fibroblast growth factor family: neuromodulation of affective behavior. Neuron 2012; 76(1): 160-174.

31. Svenningsson P, Kim Y, Warner-Schmidt J, Oh YS, Greengard P. p11 and its role in depression and therapeutic responses to antidepressants. Nat Rev Neurosci 2013; 14(10): 673-680.

32. Madureira PA, O'Connell PA, Surette AP, Miller VA, Waisman DM. The biochemistry and regulation of S100A10: a multifunctional plasminogen receptor involved in oncogenesis. J Biomed Biotechnol 2012; 2012: 353687.

33. Gordon J, Amini S, White MK. General overview of neuronal cell culture. Methods Mol Biol 2013; 1078: 1-8.

34. Beyer CE, Cremers TI. Do selective serotonin reuptake inhibitors acutely increase frontal cortex levels of serotonin? Eur J Pharmacol 2008; 580(3): 350-354.

35. Luo M, Zhou J, Liu Z. Reward processing by the dorsal raphe nucleus: 5-HT and beyond. Learn Mem 2015; 22(9): 452-460.

36. Croll SD, Chesnutt CR, Rudge JS, Acheson A, Ryan TE, Siuciak JA et al. Co-infusion with a TrkB-Fc receptor body carrier enhances BDNF distribution in the adult rat brain. Exp Neurol 1998; 152(1): 20-33.

37. Polleux F, Whitford KL, Dijkhuizen PA, Vitalis T, Ghosh A. Control of cortical interneuron migration by neurotrophins and PI3-kinase signaling. Development 2002; 129(13): 3147-3160.

38. Tapley P, Lamballe F, Barbacid M. K252a is a selective inhibitor of the tyrosine protein kinase activity of the trk family of oncogenes and neurotrophin receptors. Oncogene 1992; 7(2): 371-381.

39. Fields RD, Stevens-Graham B. New insights into neuron-glia communication. Science 2002; 298(5593): 556562.

40. von Bernhardi R, Eugenin-von Bernhardi J, Flores B, Eugenin Leon J. Glial Cells and Integrity of the Nervous System. Adv Exp Med Biol 2016; 949: 1-24.

41. Zuchero JB, Barres BA. Glia in mammalian development and disease. Development 2015; 142(22): 38053809.

42. Rajkowska G, Stockmeier CA. Astrocyte pathology in major depressive disorder: insights from human postmortem brain tissue. Curr Drug Targets 2013; 14(11): 1225-1236.

43. Rial D, Lemos C, Pinheiro H, Duarte JM, Goncalves FQ, Real Jl et al. Depression as a Glial-Based Synaptic Dysfunction. Front Cell Neurosci 2015; 9: 521.

44. Zhou X, Xiao Q, Xie L, Yang F, Wang L, Tu J. Astrocyte, a Promising Target for Mood Disorder Interventions. Front Mol Neurosci 2019; 12: 136.

45. David J, Gormley S, Mclntosh AL, Kebede V, Thuery G, Varidaki A et al. L-alpha-amino adipic acid provokes depression-like behaviour and a stress related increase in dendritic spine density in the pre-limbic cortex and hippocampus in rodents. Behav Brain Res 2019; 362: 90-102.

46. Fields RD. White matter in learning, cognition and psychiatric disorders. Trends Neurosci 2008; 31(7): 361 370.

47. Zhou B, Zhu Z, Ransom BR, Tong X. Oligodendrocyte lineage cells and depression. Mol Psychiatry 2021; 26(1): 103-117.

48. Brigadski T, Lessmann V. The physiology of regulated BDNF release. Cell Tissue Res 2020; 382(1): 15-45.

49. Fulmer CG, VonDran MW, Stillman AA, Huang Y, Hempstead BL, Dreyfus CF. Astrocyte-derived BDNF supports myelin protein synthesis after cuprizone-induced demyelination. J Neurosci 2014; 34(24): 81868196.

50. Holt LM, Hernandez RD, Pacheco NL, Torres Ceja B, Hossain M, Olsen ML. Astrocyte morphogenesis is dependent on BDNF signaling via astrocytic TrkB.T1. Elife 2019; 8. 
51. Brumwell CL, Hossain WA, Morest DK, Bernd P. Role for basic fibroblast growth factor (FGF-2) in tyrosine kinase (TrkB) expression in the early development and innervation of the auditory receptor: in vitro and in situ studies. Exp Neurol 2000; 162(1): 121-145.

52. Kang W, Balordi F, Su N, Chen L, Fishell G, Hebert JM. Astrocyte activation is suppressed in both normal and injured brain by FGF signaling. Proc Natl Acad Sci U S A 2014; 111(29): E2987-2995.

53. Bansal R. Fibroblast growth factors and their receptors in oligodendrocyte development: implications for demyelination and remyelination. Dev Neurosci 2002; 24(1): 35-46.

54. Birey F, Kloc M, Chavali M, Hussein I, Wilson M, Christoffel DJ et al. Genetic and Stress-Induced Loss of NG2 Glia Triggers Emergence of Depressive-like Behaviors through Reduced Secretion of FGF2. Neuron 2015; 88(5): 941-956.

55. Banasr M, Dwyer JM, Duman RS. Cell atrophy and loss in depression: reversal by antidepressant treatment. Curr Opin Cell Biol 2011; 23(6): 730-737.

56. Zhou X, Franklin RA, Adler M, Jacox JB, Bailis W, Shyer JA et al. Circuit Design Features of a Stable TwoCell System. Cell 2018; 172(4): 744-757 e717.

57. Katz MM, Tekell JL, Bowden CL, Brannan S, Houston JP, Berman N et al. Onset and early behavioral effects of pharmacologically different antidepressants and placebo in depression. Neuropsychopharmacology 2004; 29(3): 566-579.

58. Stassen HH, Delini-Stula A, Angst J. Time course of improvement under antidepressant treatment: a survivalanalytical approach. Eur Neuropsychopharmacol 1993; 3(2): 127-135.

59. Nierenberg AA, Farabaugh AH, Alpert JE, Gordon J, Worthington JJ, Rosenbaum JF et al. Timing of onset of antidepressant response with fluoxetine treatment. Am J Psychiatry 2000; 157(9): 1423-1428.

60. Siddiqi SH, Taylor SF, Cooke D, Pascual-Leone A, George MS, Fox MD. Distinct Symptom-Specific Treatment Targets for Circuit-Based Neuromodulation. Am J Psychiatry 2020; 177(5): 435-446.

61. Gao Z, Lee P, Stafford JM, von Schimmelmann M, Schaefer A, Reinberg D. An AUTS2-Polycomb complex activates gene expression in the CNS. Nature 2014; 516(7531): 349-354.

62. Kriaucionis $\mathrm{S}$, Heintz $\mathrm{N}$. The nuclear DNA base 5-hydroxymethylcytosine is present in Purkinje neurons and the brain. Science 2009; 324(5929): 929-930.

63. Stuart T, Butler A, Hoffman P, Hafemeister C, Papalexi E, Mauck WM, 3rd et al. Comprehensive Integration of Single-Cell Data. Cell 2019; 177(7): 1888-1902 e1821.

64. Zappia L, Oshlack A. Clustering trees: a visualization for evaluating clusterings at multiple resolutions. Gigascience 2018; 7(7).

65. Neubeck A, Van Gool L. Efficient Non-Maximum Suppression. 18th International Conference on Pattern Recognition (ICPR'06) 2006; 3: 850-855. 
bioRxiv preprint doi: https:Hdei.org/10.1101/2021.07.23.453443; this version posted July 25,2021 . The copyright holder for this preprint (which

A

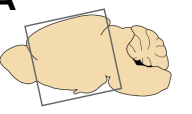

Mouse cerebral cortex

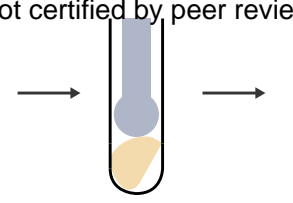

Tissue homogenization

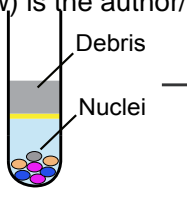

Gradient

Centrifugation

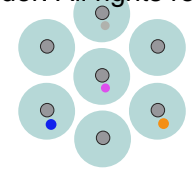

single nuclei 10x barcoding and RNASeq

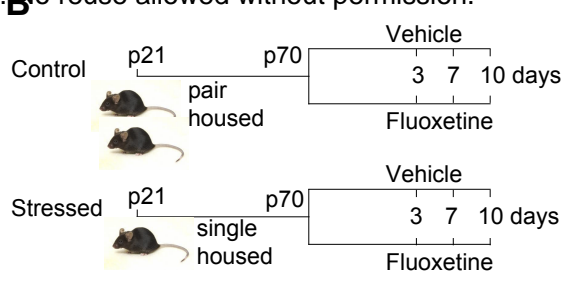

C

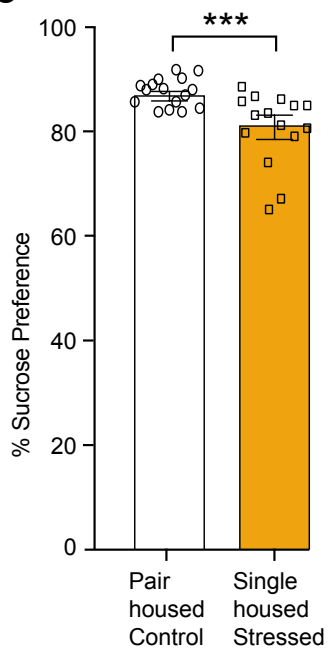

D

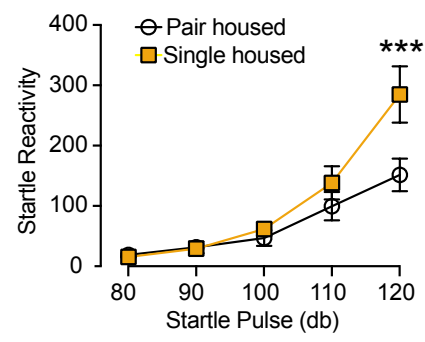

$\mathbf{F}$

Comparison of differentially expressed genes

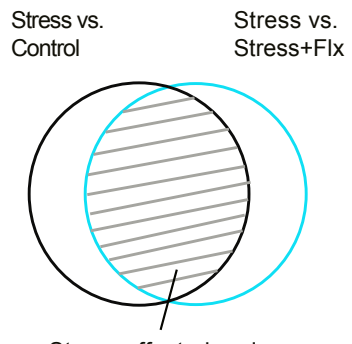

Stress-affected and

Flx normalized genes
E

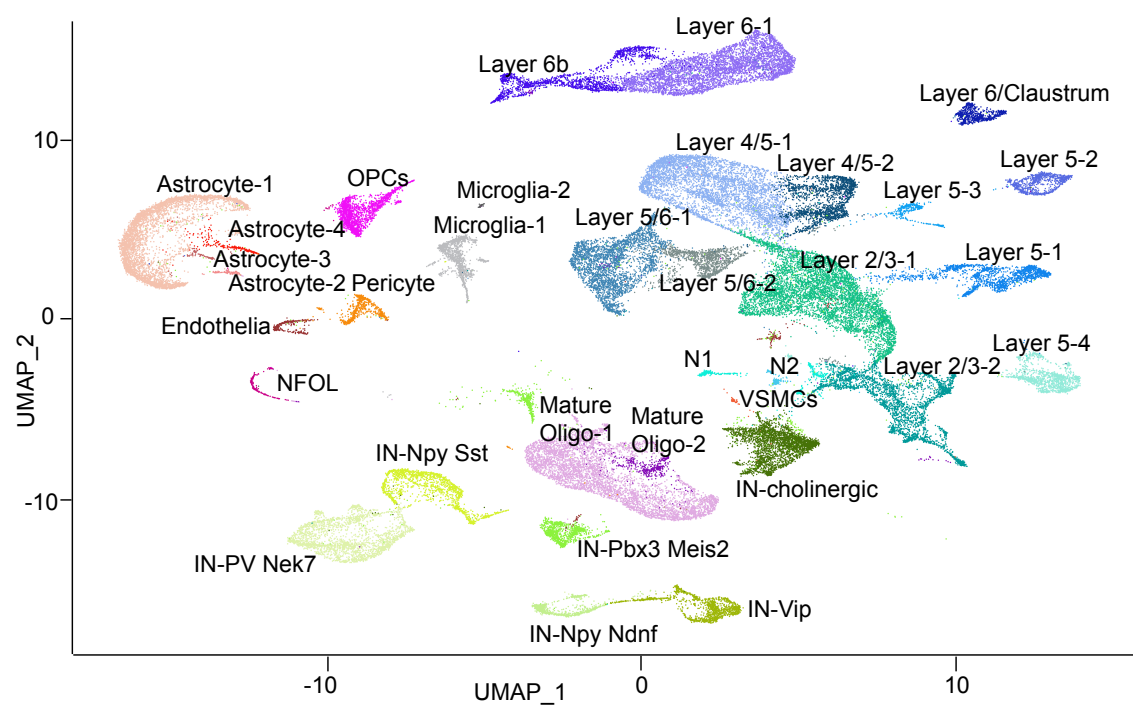

G

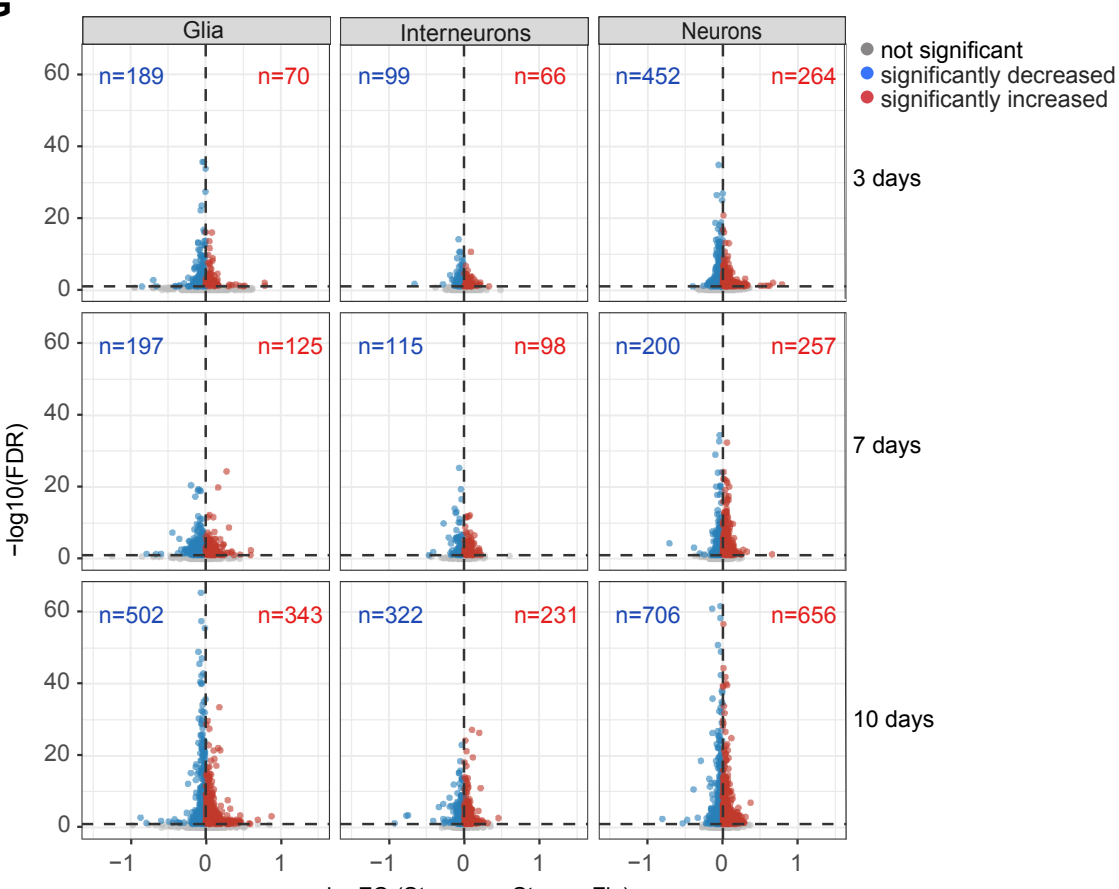

$\log \mathrm{FC}$ (Stress vs. Stress+FIx)

Figure 1. Single nuclei RNA-sequencing analysis of the mouse cortex in response to stress and fluoxetine treatment. (A) Mouse cerebral cortex was homogenized, subjected to gradient centrifugation, nuclei purification, single nuclei 10X barcoding, and RNA sequencing. (B) Mice were subjected to social isolation rearing stress by single housing from postnatal days P21 until P70, and control mice were pair-housed. Both cohorts were treated with fluoxetine (FIx) for days 3 , 7 , and $10, n=6$ samples each for four treatment groups of Control, ControlFIx, Stress, StressFlx for 3 time points. For each condition, nuclei from $n=6$ animals were pooled. (C) Sucrose preference test (SPT) on control and stressed cohorts to measure anhedonia to assess depressive-like behavior, $n=16$, pair housed; $n=15$, single housed on days P64 to P66. (D) Acoustic startle response to test reflexive behavior as a readout of emotional state, $n=16$, pair housed; $n=15$, single housed on day P68. (E) Uniform manifold approximation and projection (UMAP) plot of mouse cortical cells from pooled $n=6$ samples each of Control, ControlFIx, Stress, StressFIx for 3 time points. Cell cluster labels were annotated based on cell type specific expression of marker genes. Multiple layer subtypes likely representing the same layers from different sub-regions of the cortex were labeled with additional numbers (e.g., Layer 2/3-1 and Layer 2/3-2). Two ungrouped small populations of neurons were called N1 and N2. We identified five inter-neuronal (IN) populations. Vascular smooth muscle cells (VSMCs), two microglial, an endothelial, a pericyte, and four astrocyte populations were identified. Four oligodendrocyte clusters: Oligodendrocyte precursor cells (OPCs); newly formed oligodendrocytes (NFOL); and two populations of mature oligodendrocytes were identified. (F) Venn diagram describing the identification of stress-affected Flx-normalized genes by overlaying the differentially expressed genes from the chronically stressed versus control and the chronically stressed versus chronically stressed and treated with fluoxetine comparisons from all cell types. (G) Volcano plots showing differential gene expression for stress-affected fluoxetine-normalized genes in the glial, inter-neuronal, and neuronal populations at 3, 7,10 days (shown for one comparison StressFlx versus stress). Genes significantly upregulated by stress and downregulated by Flx (blue dots), genes downregulated by stress and upregulated by Flx (red dots), and not significant genes (grey dots) are shown. For statistical analysis in C, comparisons were made between pair-housed and single-housed mice $(n=15-16)$ using unpaired t-test with Welch's correction. In $\mathbf{D}$, comparisons were made between pair-housed and single-housed mice $(n=15-16)$ that received various intensities of startle pulse using two-way ANOVA and corrections for multiple comparisons was done using Bonferroni's multiple comparisons test. Data are mean $+/-\mathrm{SEM} ;{ }^{*} P \leq 0.05,{ }^{* *} P \leq 0.01,{ }^{* * *} P \leq 0.005,{ }^{* * *} P \leq 0.001$. 
bioRxiv preprint doi: https://doi.org/10.1101/2021.07.23.453443; this version posted July 25, 2021. The copyright holder for this preprint (which A was not certified by peer review) is the author/funder. All rights reserved. No reuse allowed without permission.
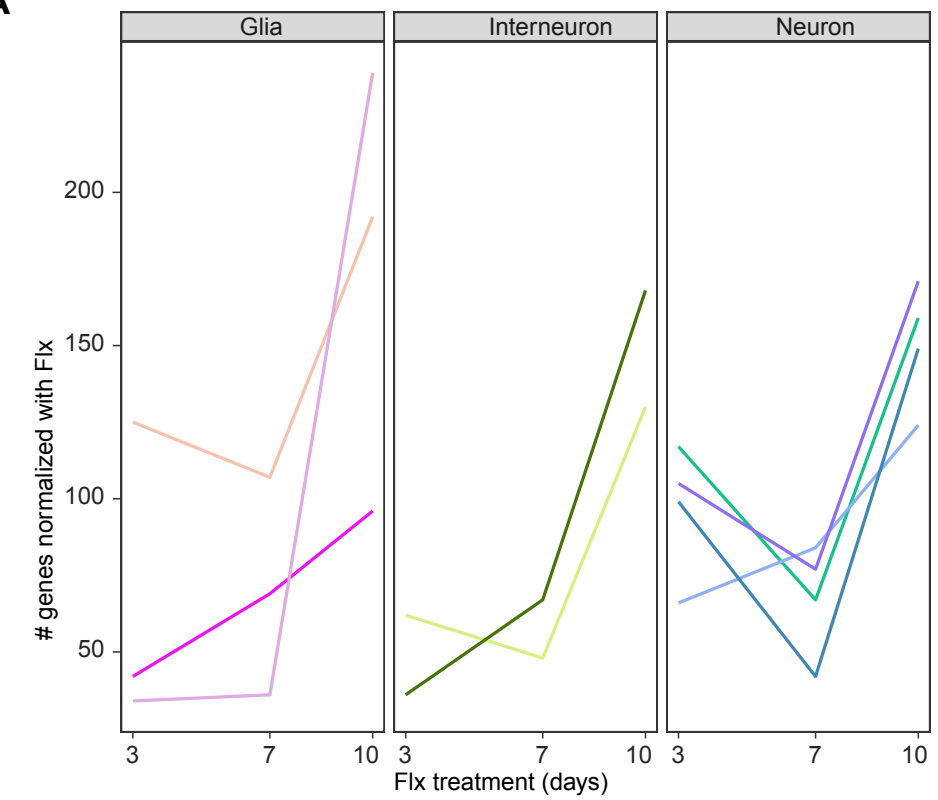

- Oligodendrocyte precursor cells (OPCs)

- Mature Oligodendrocytes

- PV Interneurons

- Cholinergic interneurons

Layer 2/3-1 Neurons

- Layer 4/5a-1 Neurons

Layer 5/6-1 Neurons

$\mathbf{B}$

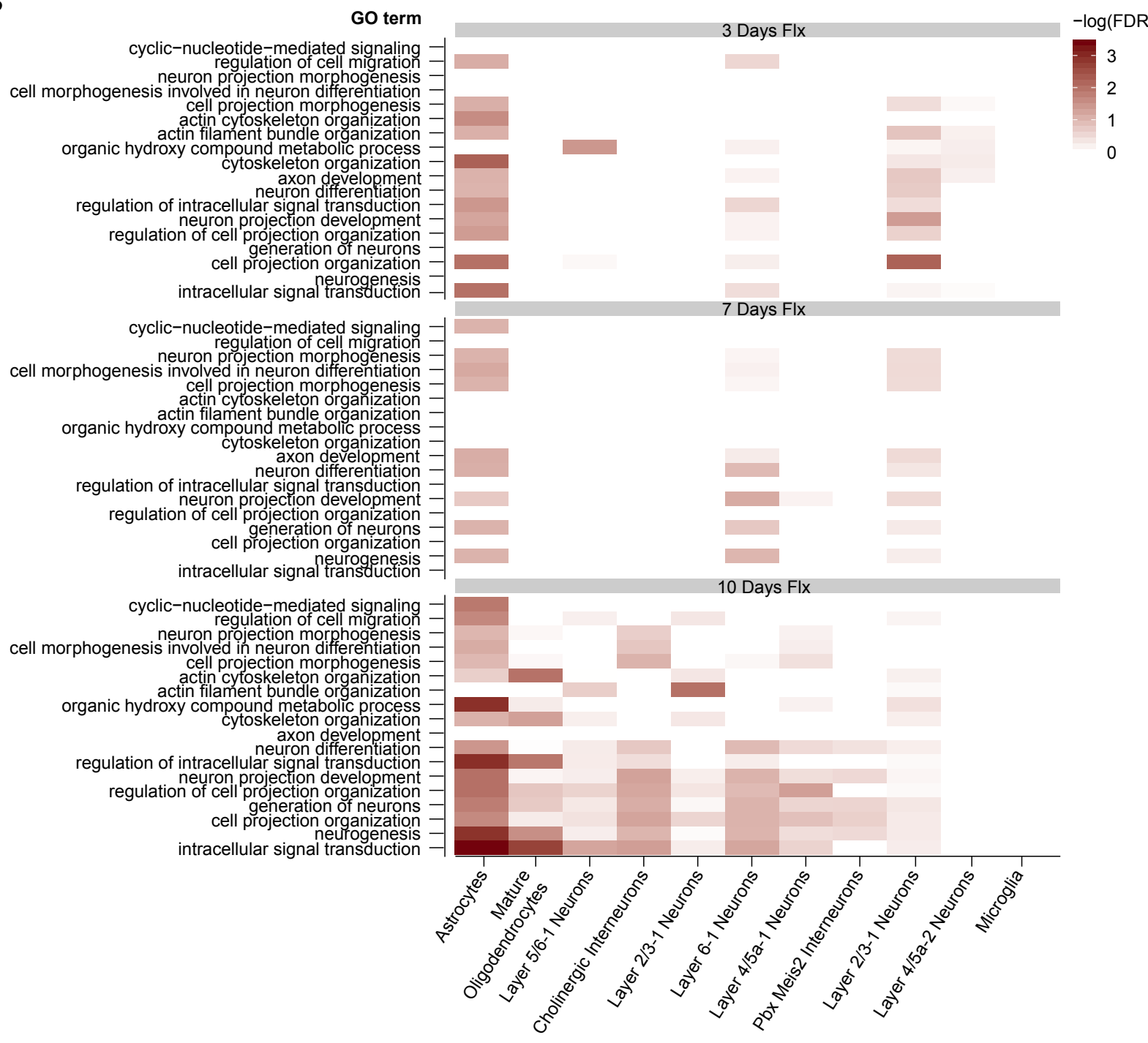

Figure 2. Transcriptional changes in neuronal and glial cell clusters in response to stress and fluoxetine treatment. (A) Kinetics of Flx-induced changes in the glial, inter-neuronal, and neuronal cell populations on days 3, 7, and 10 of treatment. Analysis was based on the stress-affected fluoxetine-normalized genes. (B) Gene ontology enrichment of the stress-affected fluoxetinenormalized genes on days 3,7 , and 10 of treatment in various cell populations. Analysis based on pooled $n=6$ samples each of Control, ControlFIx, Stress, and StressFIx at 3, 7, and 10 days of fluoxetine treatment (Extended Table 3). 
bioRxiv preprint doi: https://doi.org/10.1101/2021.07.23.453443; this version posted July 25, 2021. The copyright holder for this preprint (which was not certified by peer review) is the author/funder. All rights reserved. No reuse allowed without permission.
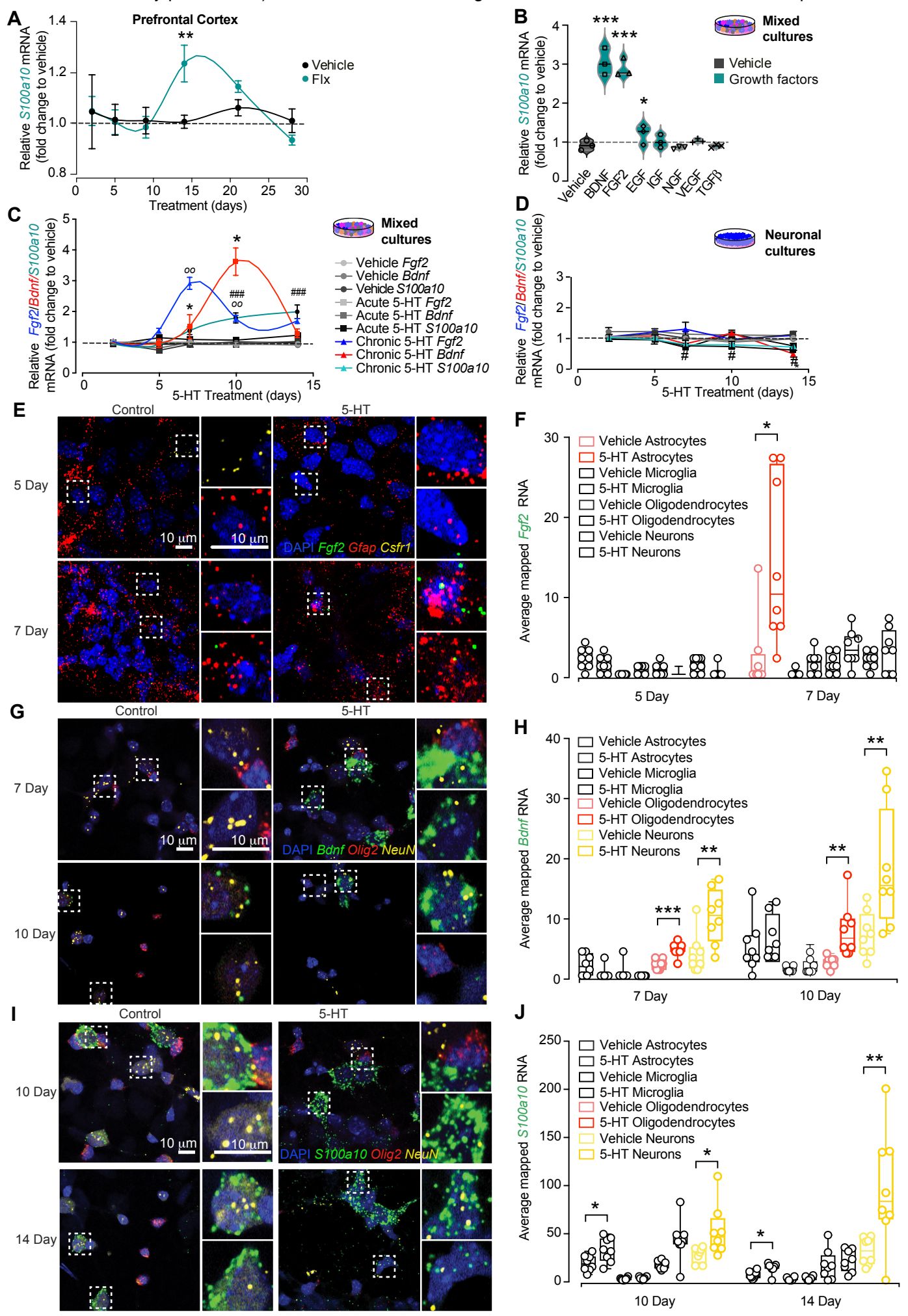

$\mathbf{J}$

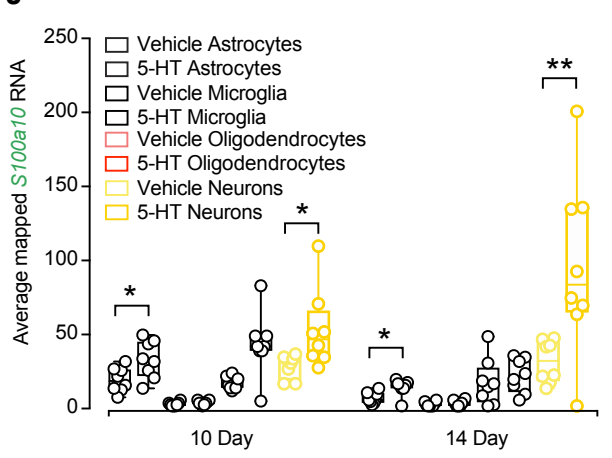

Figure 3. Reproducing the molecular response to fluoxetine in a cortical primary culture system. (A) Dynamics of $S 100 a 10$ mRNA transcription in the prefrontal cortex (PFC) by quantitative reverse-transcriptase polymerase chain reaction (qRT-PCR) at 2, 5, 7, 14, 21, and 28 days of $F / x$ treatment ( $\mathrm{n}=3$ ). (B) Analysis of S100a10 mRNA transcription in primary cortical mixed cultures by qRT-PCR upon stimulation with growth factors BDNF, FGF2, EGF, IGF, NGF, VEGF, TGF $\beta$ for $12 \mathrm{~h}(\mathrm{n}=6)$. (C) Analysis of Fgf2, Bdnf, and S100a10 mRNA transcription by qRT-PCR at 2, 5, 7, 10, 14 days upon stimulation with vehicle, acute and chronic serotonin (5-HT) treatment in primary cortical mixed cultures ( $\mathrm{n}=6)$. (D) Analysis of Fgf2, Bdnf, and S100a10 mRNA transcription by qRT-PCR at 2, 5, 7, 10, 14 days upon stimulation with vehicle, acute and chronic 5-HT treatment in primary cortical neuronal cultures $(n=6)$. (E) Fluorescent in situ hybridization using RNAscope fluorescent multiplex assay (RNA-ISH) in mixed cortical cultures simultaneously labeled with RNA probes to locate Fgf2 RNA (green) in astrocytes (using probe for Gfap RNA, red) or microglia (Csfr1 RNA, yellow). (F) Quantification of the averaged mapped Fgf2 RNA in neurons, astrocytes, oligodendrocytes, and microglia. (G) RNA-ISH in mixed cortical cultures simultaneously labeled with RNA probes to locate Bdnf RNA (green) in neurons (Rbfox3, NeuN RNA, yellow) or Oligodendrocytes (Olig2 RNA, red). (H) Quantification of the averaged mapped Bdnf RNA in neurons, astrocytes, oligodendrocytes, and microglia. (I) RNA-ISH in mixed cortical cultures simultaneously labeled with RNA probes to locate S100a10 RNA (green) in neurons (using probe for Rbfox3, NeuN RNA, yellow) or oligodendrocytes (using Olig2 RNA, red). (J) Quantification of the averaged mapped S100a10 RNA in the neuronal and glial cell types. For statistical analysis, comparisons were made between vehicle and chronic Flx (A) or between vehicle and acute or chronic serotonin (5-HT) treated samples $(\mathbf{C}$ and $\mathbf{D})$, all $(n=3)$, using two-way ANOVA and corrections for multiple comparisons were performed by running post hoc Tukey's multiple comparisons test. In $\mathbf{C}$, and $\mathbf{D}$, $p$-value significance indicated by symbols $\mathbf{0 0}$ for Fgf2, * for Bdnf, \# for S100a10. In B, comparisons were made between vehicle and growth factor-treated samples (n=3) using one-way ANOVA and corrections for multiple comparisons were done using Bonferroni multiple comparisons test. For statistical analysis in $\mathbf{F}$, $\mathbf{H}$, and $\mathbf{J}$, comparisons were made between vehicle and chronic 5-HT-treated sections $(n=8)$ for each pair of probes using two-way ANOVA to test effects of 5-HT treatment and time. Corrections for multiple comparisons were performed by running post hoc Tukey's multiple comparisons test. Data are mean $+/-\mathrm{SEM} ;{ }^{*} \mathrm{P} \leq 0.05,{ }^{* *} \mathrm{P} \leq 0.01,{ }^{* * *} \mathrm{P} \leq 0.005,{ }^{* * * *} \mathrm{P} \leq$ 0.001. Experiments were independently reproduced three times with identical results. Scale bar, $10 \mu \mathrm{m}$. 
bioRxiv preprint doi: https://doi.org/10.1101/2021.07.23.453443; this version posted July 25, 2021. The copyright holder for this preprint (which

\section{A} was not certified by peer review) is the author/funder. All rights reserved. No reuse allowed without permission.
Mixed
Neuronal
cultures
cultures
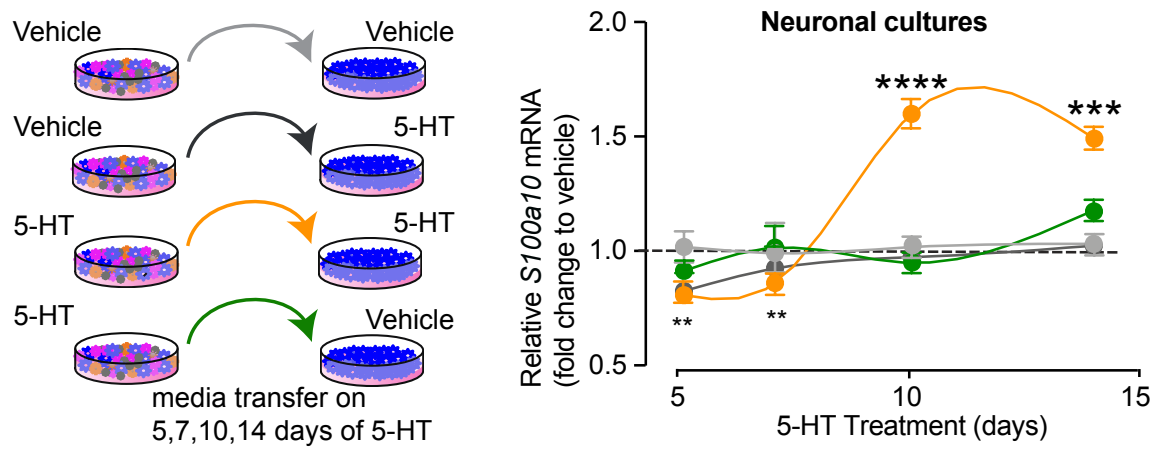

Vehicle-treated mixed-media into vehicle-treated neurons

Vehicle-treated mixed-media into 5-HT-treated neurons

5-HT-treated mixed-media into 5-HT-treated neurons

- 5-HT-treated mixed-media into vehicle-treated neurons

\section{$\begin{array}{ll}\text { Cixed } & \begin{array}{l}\text { Neuronal } \\ \text { cultures }\end{array} \\ \text { cultures }\end{array}$}

D

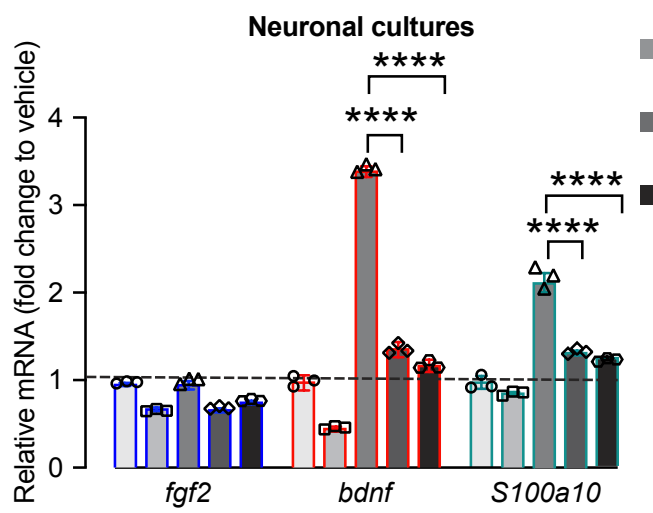

fgf2
Vehicle-treated mixed-media into vehicle-treated neurons

5-HT-treated mixed-media into vehicle-treated neurons

5-HT-treated mixed-media into 5-HT-treated neurons

5-HT-treated BDNF-depleted mixed -media into 5-HT treated neurons

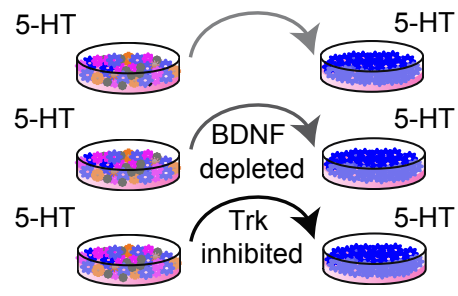

media transfer on day 10 of 5 -HT treatment

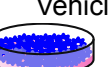

Vehicle

5-HT

5-HT

$5-\mathrm{HT}$ 5-HT-treated mixed-media into Trk-inhibited 5-HT-treated neurons

Figure 4. Neuron-glia secreted factor confers downstream serotonin-dependent effects in neurons. (A) Schematic showing media transfer from 5, 7, 10, 14 days of vehicle (light grey and black arrows) and chronic 5-HT-treated (orange and green arrows) cortical mixed cultures into 5, 7, 10, 14 days of vehicle (light grey and green circles) and chronic 5-HT-treated (black and orange circles) cortical neuronal cultures. (B) Analysis of S100a10 mRNA expression in cortical neuronal cultures by qRT-PCR after media swap from chronic 5-HT-treated cortical mixed cultures to chronic 5-HT-treated cortical neuronal cultures ( $n=3$ ). (C) Schematic showing media transfer from day 10 of vehicle and chronic 5-HT-treated cortical mixed cultures into day 10 of neuronal cultures of vehicle (two light grey bars), chronic 5-HT-treated (medium grey bar), chronic 5-HT-treated and BDNF-depleted before swap (dark grey bar), and chronic 5-HT-treated Trk autophosphorylation-inhibited (black bar) cortical neuronal cultures. (D) Analysis of Fgf2, Bdnf, and S100a10 mRNA expression in cortical neuronal cultures after media swap from chronic 5-HT-treated cortical mixed cultures to chronic 5-HT-treated cortical neuronal cultures $(n=3)$. For statistical analysis in $\mathbf{B}$, comparisons were made between vehicle and acute or chronic fluoxetine or 5-HT treated samples $(n=3)$ using two-way ANOVA and corrections for multiple comparisons were performed by running post hoc Tukey's multiple comparisons test. In D, comparisons were done using one-way ANOVA, and tested for multiple comparisons using post-hoc Bonferroni multiple comparisons test $(n=3)$. Only comparisons between last three conditions are shown. Data are mean $+/-\mathrm{SEM} ;{ }^{*} P \leq 0.05,{ }^{* \star} P \leq 0.01,{ }^{* \star *} P \leq 0.005$, ${ }^{\star \star * \star} P \leq 0.001$. Experiments were independently reproduced three times. 
bipRxiv preprint doi: https:/tedoi.org/10.1101/2021.07.23.453443; this version posted July 25,2021 . The copyright holder for this preprint (which A was not certified by peer review) is the author/funder. All rights reserved. No reuse allowed without permission.

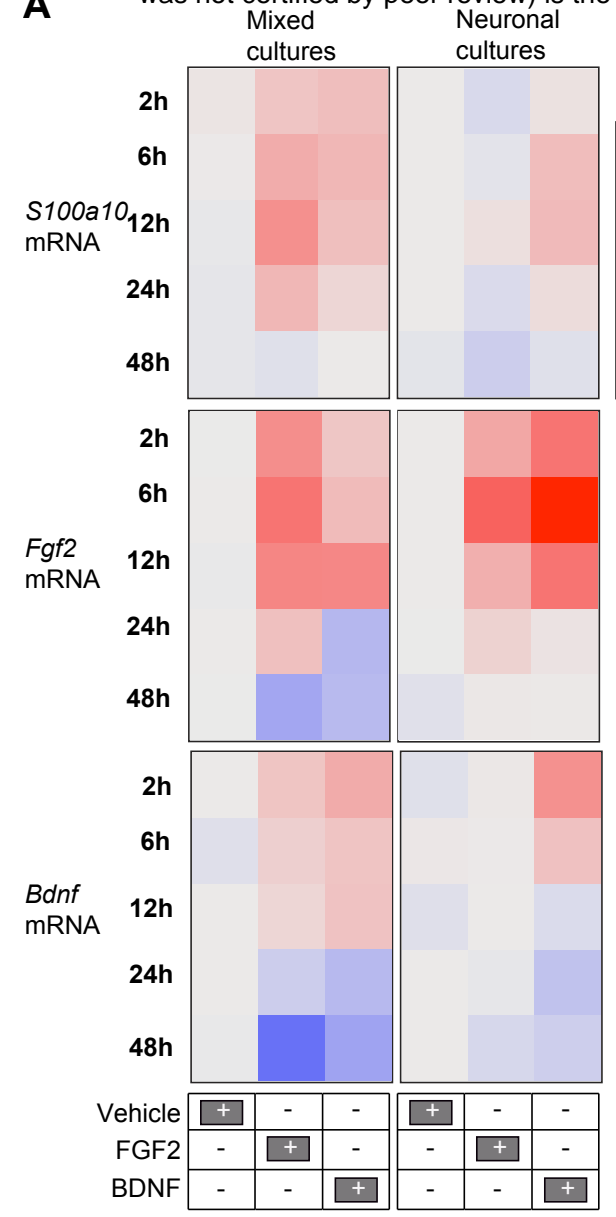

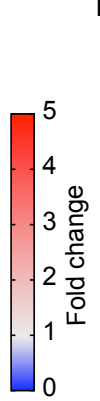

。

(2)

$$
\text { inhFr }
$$
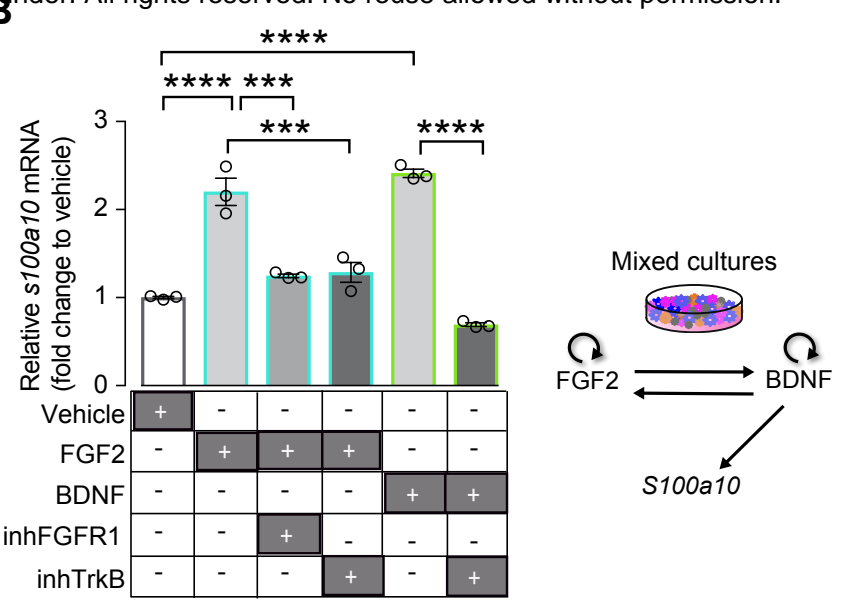

C

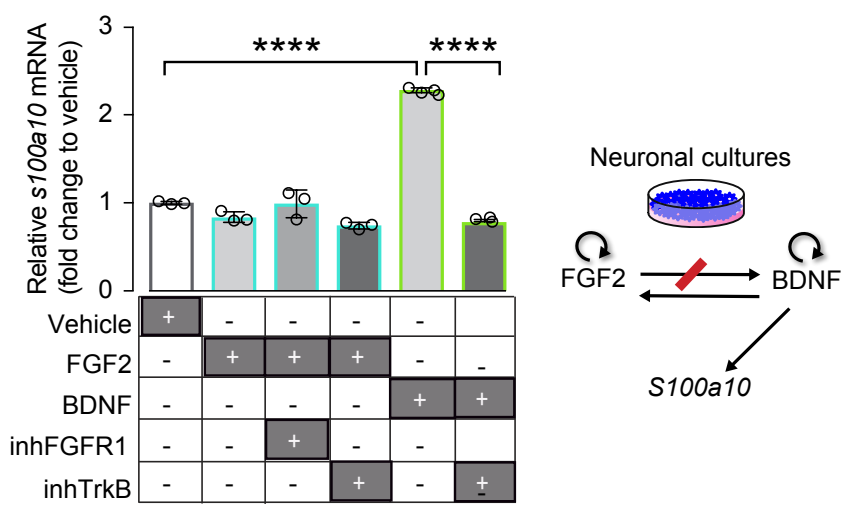

D

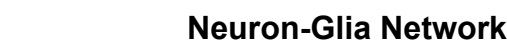

Neurons

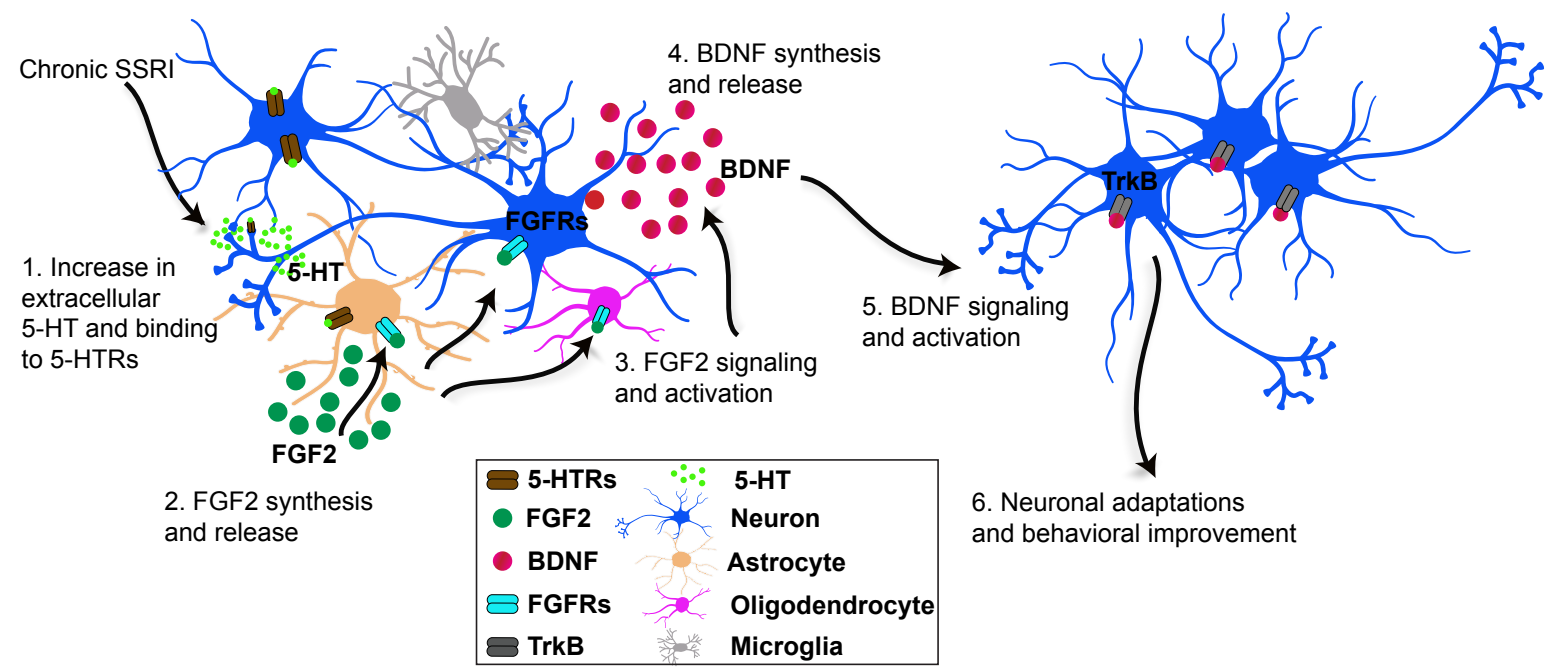

Figure 5. FGF2 regulates $B d n f$ transcription regulating reciprocal neuron-glia interactions. (A) Heatmap showing the kinetics of S100a10, Fgf2, and Bdnf mRNA expression at 2, 6, 12, 24, 48h upon stimulation with vehicle, acute FGF2 and acute BDNF in cortical mixed and cortical neuronal cultures $(n=3)$ based on qRT-PCR analysis shown in Extended figure 6. (B) Analysis of S100a10 mRNA expression by qRT-PCR at $8 \mathrm{~h}$ upon stimulation with vehicle, acute FGF2, acute BDNF, inhibition of FGFR1 (inhFGFR1) 30 min prior to acute FGF2 stimulation, and inhibition of Trk autophosphorylation (inhTrk) $30 \mathrm{~min}$ prior to acute BDNF stimulation in cortical mixed cultures $(n=3)$. Schematic showing the interaction between FGF2, BDNF, and S100a10 in cortical mixed cultures. (C) Analysis of S100a10 mRNA expression by qRT-PCR at 8 h of vehicle, acute FGF2, acute BDNF, inhibitor for FGFR1 (inhFGFR1) + acute FGF2 treatment, and inhibitor for TrkB (inhTrkB) + acute FGF2 treatment or acute BDNF treatment in cortical neuronal cultures ( $n=3$ ). Schematic showing the interaction between FGF2, BDNF, and S100a10 in cortical neuronal cultures. (D) Simplified overview showing how activation of sequential molecular signaling events make up the neuron-glia reciprocal network during chronic serotonin or fluoxetine treatment. For statistical analysis in $\mathbf{A}$, comparisons were made between vehicle and acute FGF2 or BDNF-treated samples ( $n=3$ ) using two-way ANOVA and corrections for multiple comparisons were performed by running post hoc Tukey's multiple comparisons test. In B and C, comparisons were made using one-way ANOVA, and multiple comparisons was tested using post-hoc Bonferroni test. Data are mean $+/-$ SEM; ${ }^{*} P \leq 0.05,{ }^{* *} P \leq 0.01,{ }^{* * *} P \leq 0.005,{ }^{* * *} P \leq 0.001$. Experiments were independently reproduced three times with identical results. 
bioRxiv preprint doi: https://doi.org/10.1101/2021.07.23.453443; this version posted July 25, 2021. The copyright holder for this preprint (which

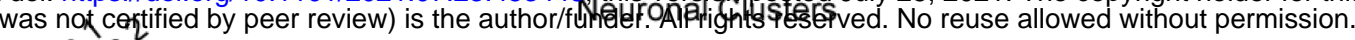

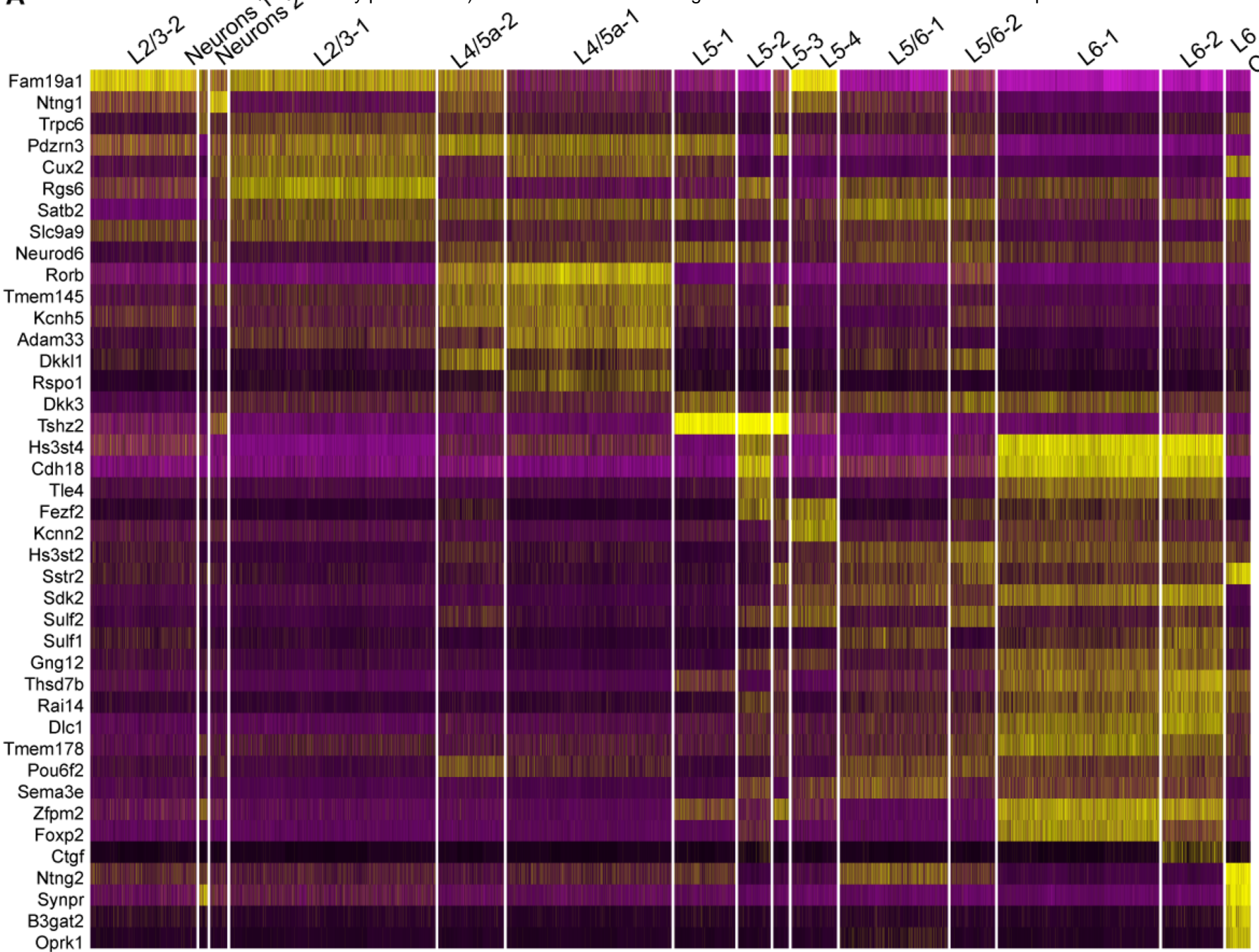

Oprk1

Non-Neuronal Clusters
C

Interneuronal Clusters

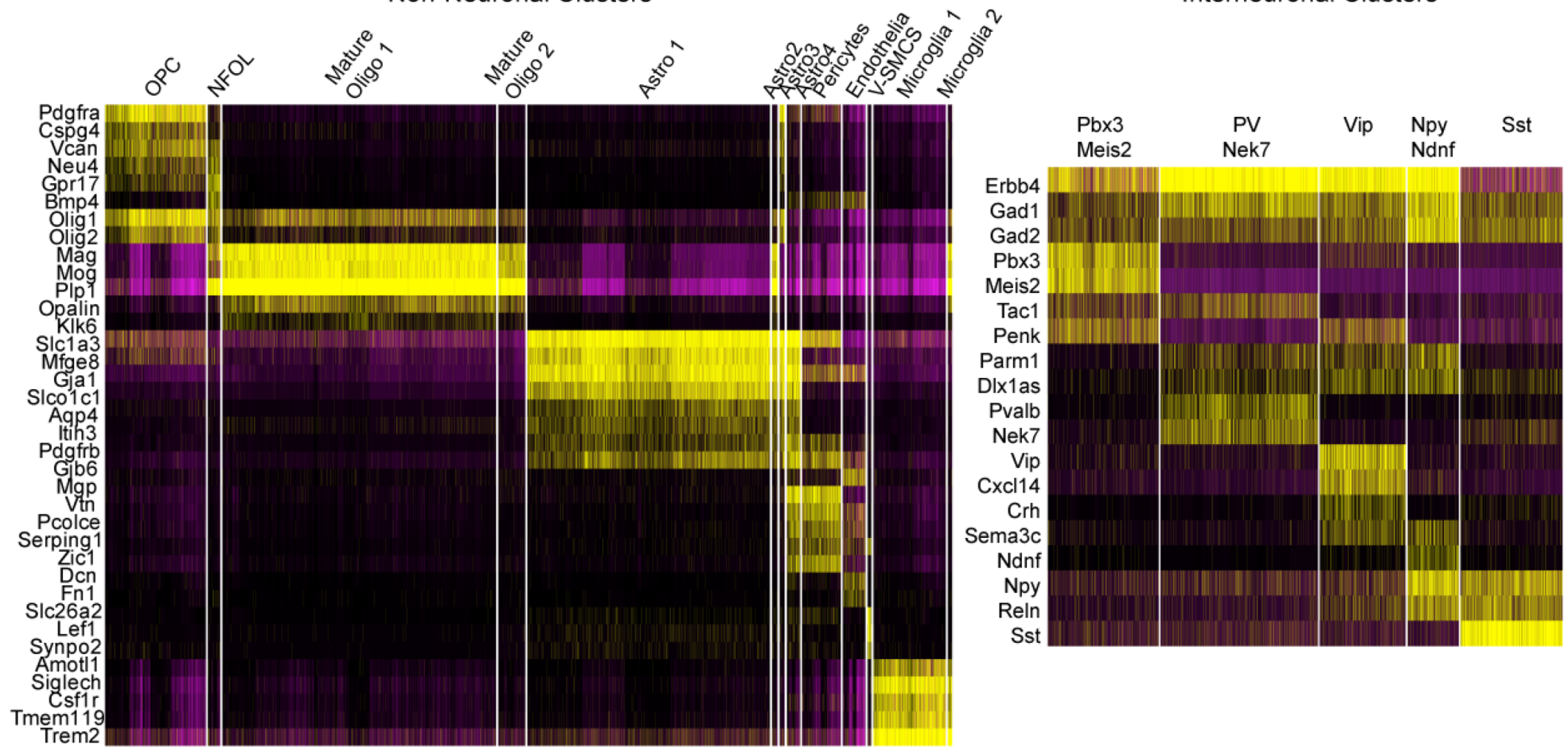

Extended Figure 1. Classification of neuronal, non-neuronal and inter-neuronal cell type clusters based on cell type specific marker gene expression. (A) Heatmap showing the expression of cell-type specific marker genes in the cortical neuronal clusters. Cortical neurons are assigned layer-specific identity based on the expression of layer-specific marker genes. Each row represents the expression of a single gene, and each column represents a single nuclei from a single cell, and many such cells with similar gene expression profiles are clustered and grouped. (B) Heatmap showing the expression of cell type specific marker genes in the cortical non-neuronal clusters. (C) Heatmap showing the expression of cell type specific marker genes in the inter-neuronal clusters. Samples are $\mathrm{n}=12 \mathrm{comprising}$ four treatment groups of control, controlFIx, stress, stressFlx at 3 time points. For each time point, for each treatment group, nuclei from $n=6$ animals were pooled. 
bioRxiv preprint doi: https://doi.org/10.1101/2021.07.23.453443; this version posted July 25, 2021. The copyright holder for this preprint (which

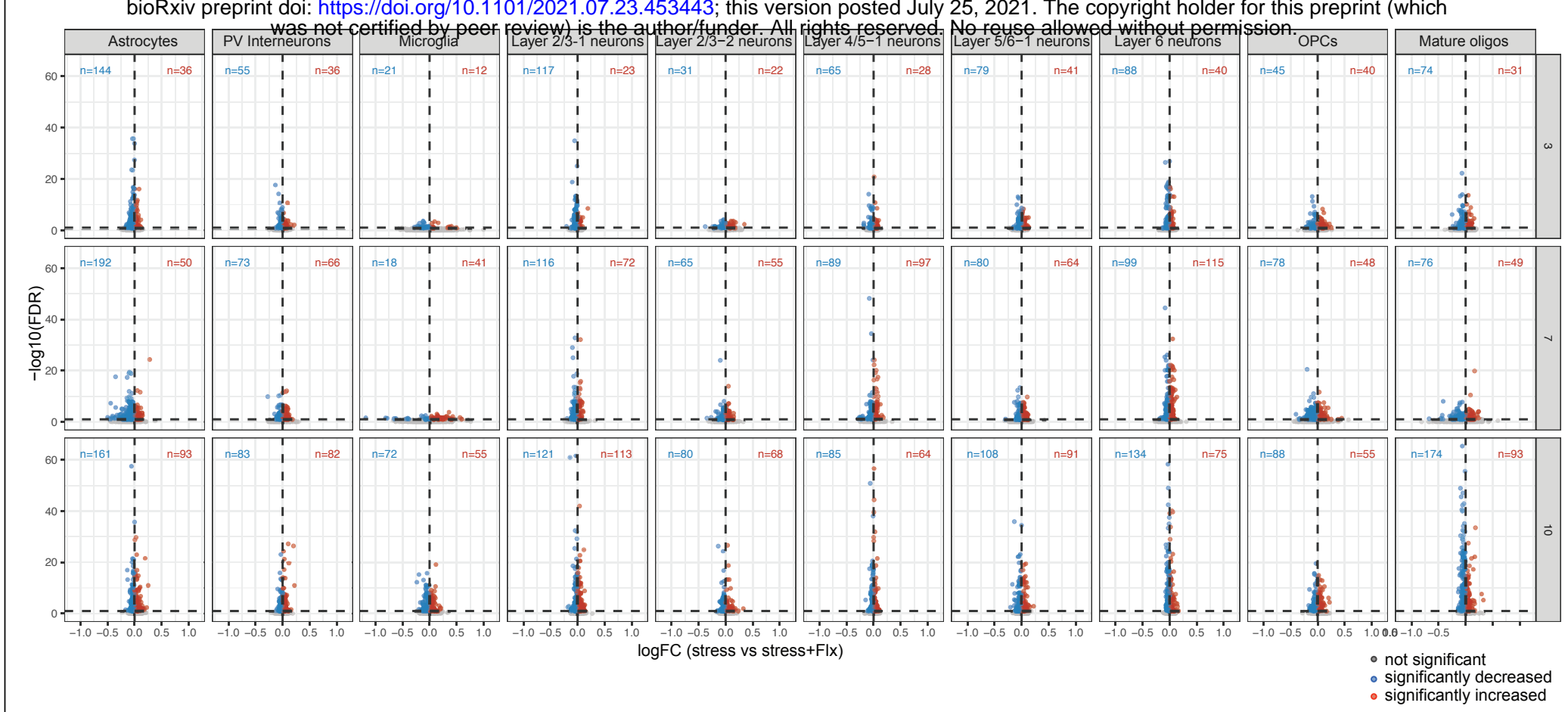

Extended Figure 2. Volcano plots showing the differential expression of stress-affected fluoxetine-normalized genes. Differential gene expression for stressaffected fluoxetine-normalized genes in the glial, inter-neuronal, and neuronal populations at 3, 7, 10 days (shown for one comparison of Stress versus StressFlx). Genes significantly upregulated by stress and downregulated by Flx (blue dots), genes downregulated by stress and upregulated by Flx (red dots), and not significant genes (grey dots) are shown. Genes that are significantly differentially expressed in both conditions (FDR $<0.1$ ), and changing in opposite directions (logFC $>0$ ) in stress versus control and $(\log \mathrm{FC}<0)$ in fluoxetine-treated stress versus stress, or vice versa). 
A Day 3 3 FlX

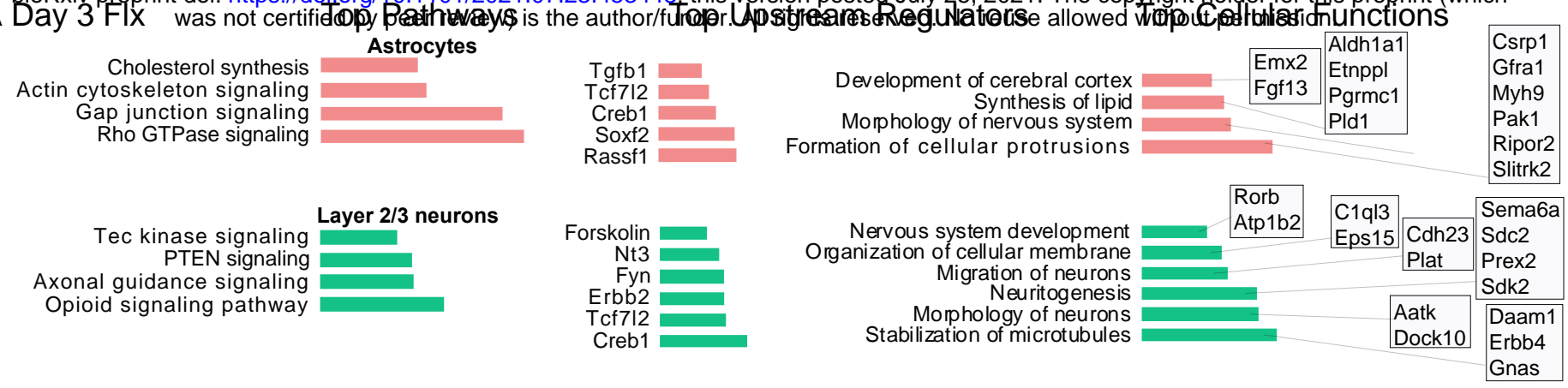

B Day 7 Flx

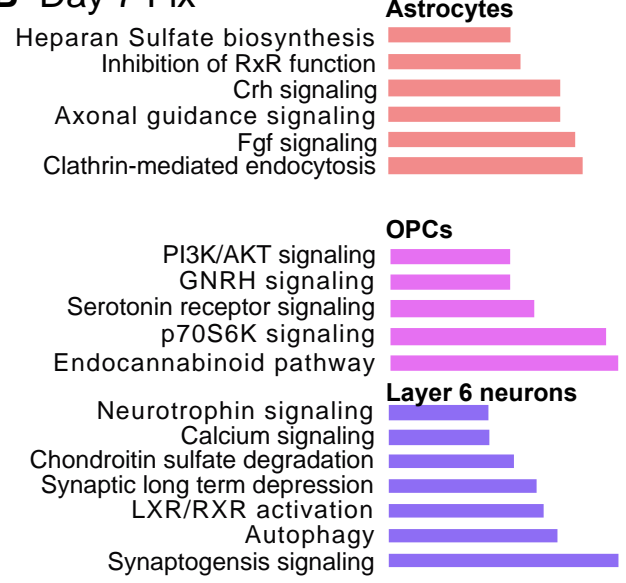

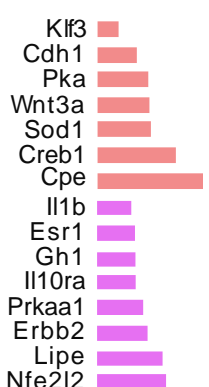

Lipe

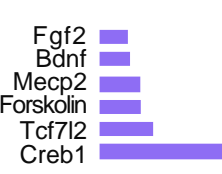

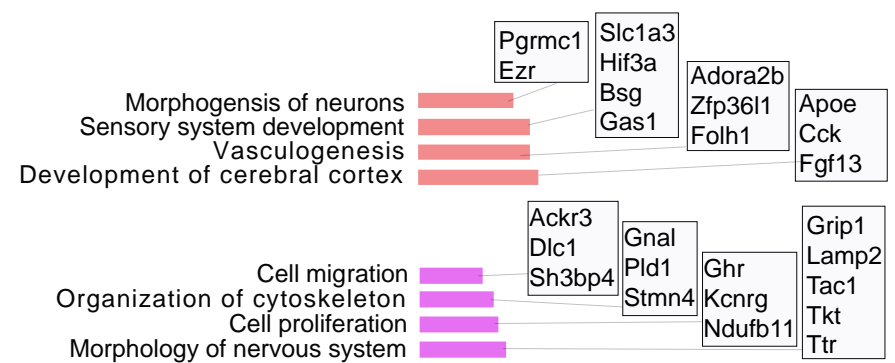

Morphology of nervous system

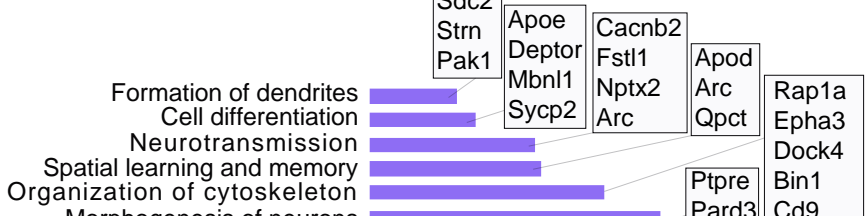

Morphogenesis of neurons

\section{Day $10 \mathrm{Flx}$}

Synaptic long term potentiation Synaptogenesis signaling pathway CAMP signaling

Ephrin receptor signaling
Axonal guidance signaling Wnt b-catenin signaling

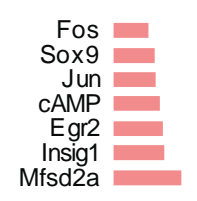

Oligodendrocytes

Ephrin receptor signaling Synaptogenesis signaling pathway
Neuregulin signaling
Actin nucleation complex
Axonal guidance signaling

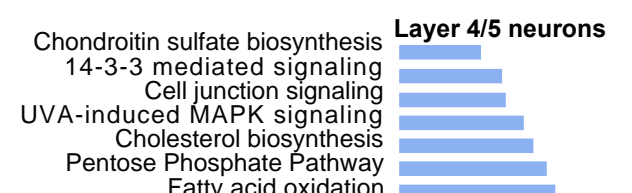

Layer 5 neurons

14-3-3 mediated signaling

Protein kinase A signaling Cell junction signaling ERK5 signaling Fatty acid oxidation
Pentose phosphate pathway

Phagosome maturation

Axonal guidance signaling ERK/MAPK signaling LXR/RXR activation Synaptogenesis signaling pathway CAMP-mediated signaling

Actin cytoskeleton signaling

VEGF signaling
Dermatan sulfate biosynthesis RhoGDI signaling Fatty acid activation Cholesterol biosynthesis Pentose phosphate pathway

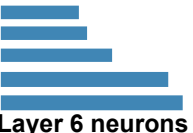

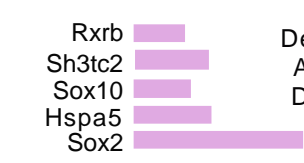

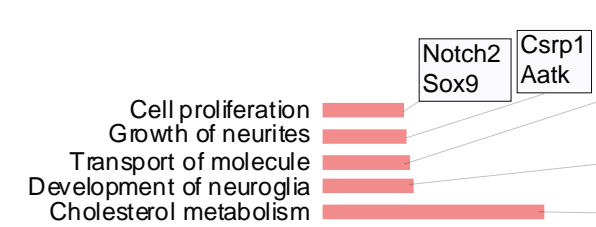

Kcnj10 Kcnj16 Grin2a Ezr Slc9a3r1 Development of neuroglia Cholesterol metabolism

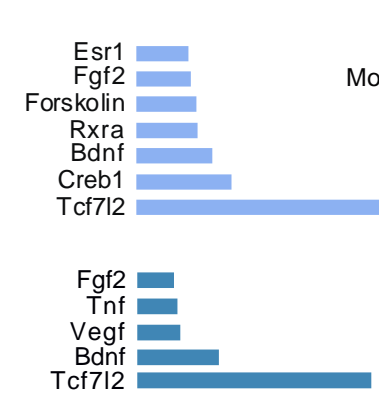

evelopmental process of synapse Assembly of intercellular junctions
Differentiation of oligodendrocytes Myelination of cells

Organization of cytoskeleton

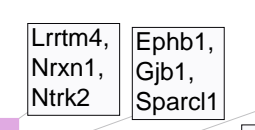

II33
Hspa8

Srebf1
Phgdh

Myrf,

Nkx6-2,

Ntrk2

\begin{tabular}{|l|l|}
\hline Mag, & Adamts4, \\
Mbp, & Daam1, \\
Ugt8, & Clu, \\
Myh14 & Epha5, \\
& Epha6, \\
\hline
\end{tabular}

\begin{tabular}{|c|c|c|c|}
\hline \multirow{4}{*}{$\begin{array}{c}\text { Morphology of cerebral cortex } \\
\text { Polarization of cells } \\
\text { Hyperpolarization }\end{array}$} & & & \multirow{3}{*}{$\begin{array}{l}\text { Bcl2l1 } \\
\text { Fstl1 } \\
\text { Npy }\end{array}$} \\
\hline & $\begin{array}{l}\text { Fgf1, } \\
\text { Lama2 }\end{array}$ & \multirow{2}{*}{$\begin{array}{l}\text { Cd81, } \\
\text { Scd2, } \\
\text { Enpp2 }\end{array}$} & \\
\hline & & & \\
\hline & \multirow{3}{*}{\begin{tabular}{l|} 
Elmo1 \\
Sgk1
\end{tabular}} & \multirow{3}{*}{\begin{tabular}{|l|} 
Inhba \\
Arhgap23
\end{tabular}} & \multirow{3}{*}{$\begin{array}{l}\text { Atp1b3 } \\
\text { Nr3c2 }\end{array}$} \\
\hline Neuritogenesis & & & \\
\hline tomenctacic of $\mathrm{Na}$ & & & \\
\hline
\end{tabular}

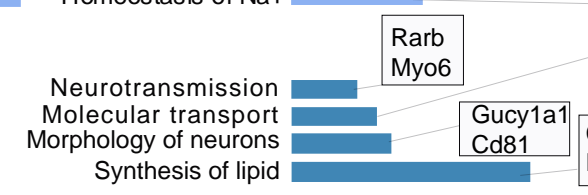

\begin{tabular}{|l|}
\hline Inhba, \\
Kif13a, \\
Mt1, \\
Atp6v0e1, \\
Ywhaq \\
\hline
\end{tabular}

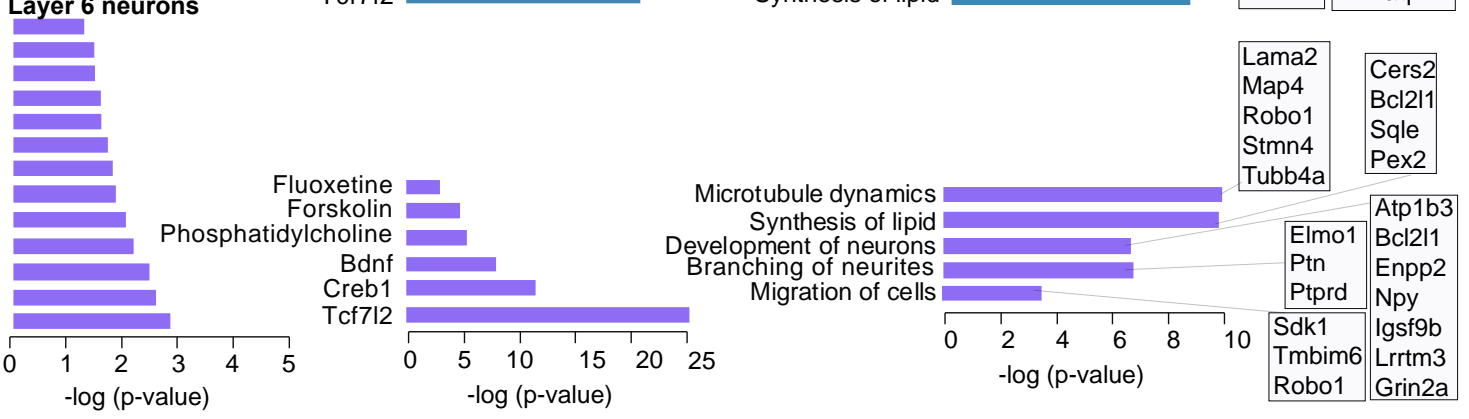

Extended Figure 3. Cell type specific molecular and cellular functions regulated by the stress-affected fluoxetine-normalized genes by Ingenuity Pathway Analysis. (A) Enriched pathways, top upstream regulators, and enriched cellular functions in astrocytes and Layer $2 / 3$ neurons on day 3 of Flx treatment. (B) Enriched pathways, top upstream regulators, and enriched cellular functions in astrocytes, OPCs, and Layer 6 neurons on day 7 of FIx treatment. (C) Enriched pathways, top upstream regulators, and enriched cellular functions in astrocytes, mature oligodendrocytes, Layer 4/5a neurons, Layer 5/6 and Layer 6 neurons on day 10 of Flx treatment. The significant values ( $p$-value of overlap) for enriched pathways and enriched cellular functions indicate the probability of association of molecules from the dataset with the canonical pathway by random chance alone and is calculated by the right-tailed Fischer's exact test and is displayed along the $x$-axis. Upstream regulator analysis identifies molecules that affect the expression, transcription or phosphorylation of genes in the experimental dataset, and the significant overlap $p$-value $(P<0.05)$ is based on right-tailed Fisher's exact test. 
bioRxiv preprint doi: https://doi.org/10.1101/2021.07.23.453443; this version posted July 25, 2021. The copyright holder for this preprint (which A was not certified by peer review) is the author/fund $\mathbf{B}$ All rights reserved. No reuse allowed without permission.
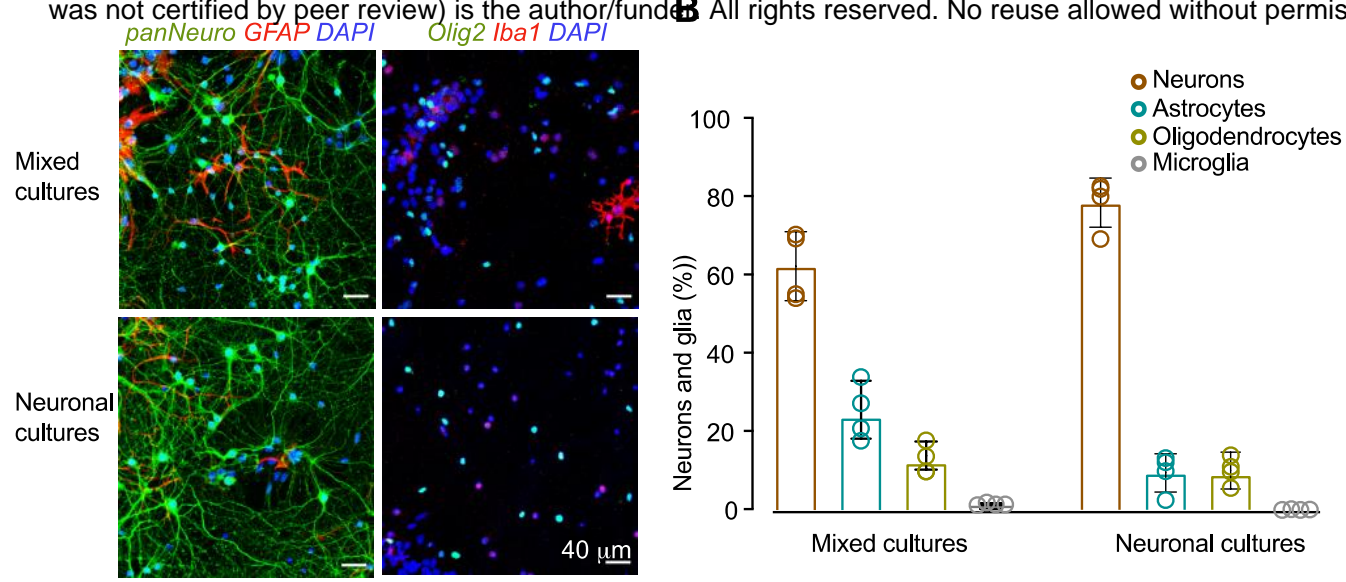

C

\section{D}
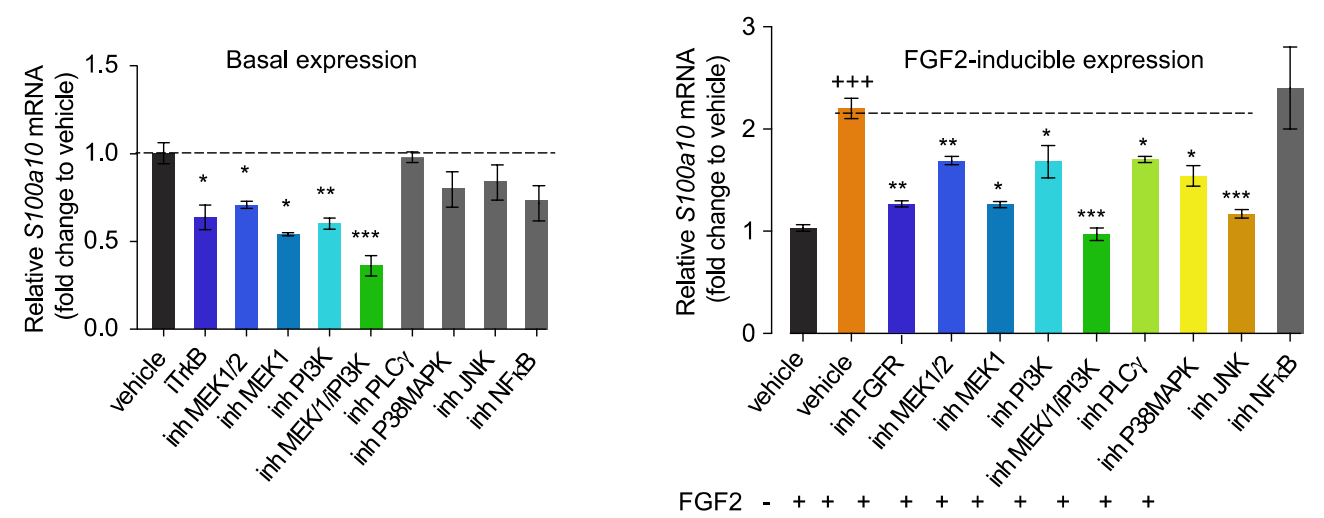

E

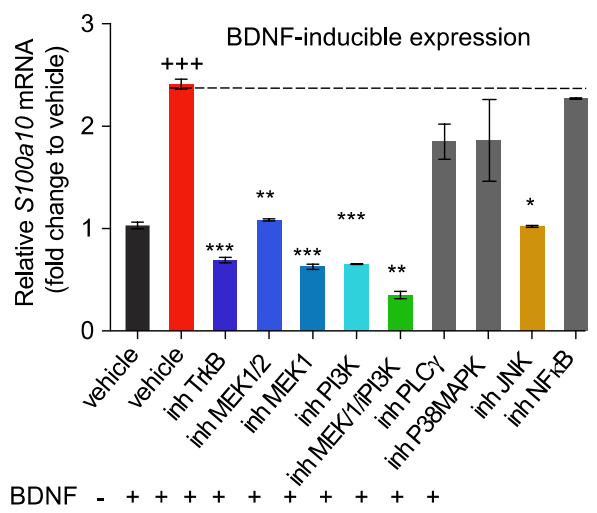

G

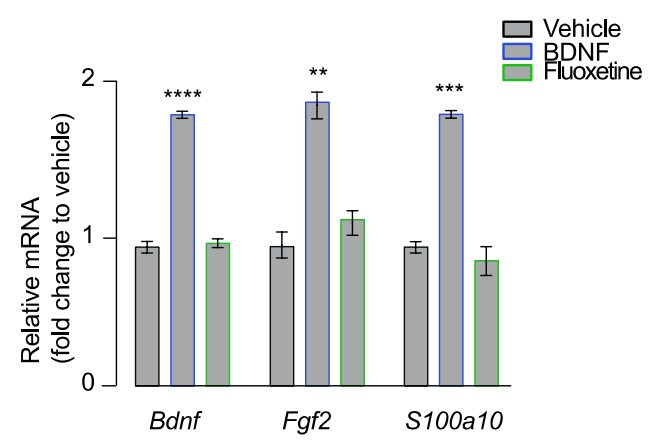

$\mathbf{F}$

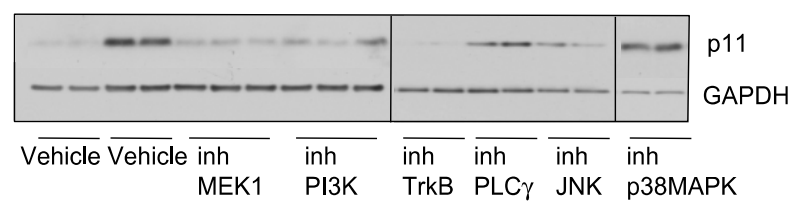

BDNF

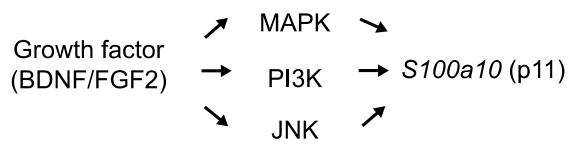

Extended Figure 4. Primary cortical culture cell types and stimulation of S100a10 mRNA by growth factors and fluoxetine. (A) Representative images of immunofluorescence analysis in DIV12 (days in vitro) primary cortical mixed and neuronal cultures to label neurons (pan-neuronal antibody), astrocytes (GFAP antibody), oligodendrocytes (Olig2 antibody), microglia (Iba1 antibody), and all nuclei (DAPI). (B) Cell counting of neuronal and glial cell types using ImageJ analysis. Cells were assigned to one of the four cell types based on the intensity of fluorescent signal from each cell marker staining. Cells were counted from $n=3$ sections, $n=3$ replicates and classified per data point. (C) basal (D), FGF2- (E), and BDNF-inducible S100a10 mRNA expression upon pharmacological inhibition of the receptor tyrosine kinase pathways in cortical mixed cultures. Inhibitors for TrkB (K252a), FGFRs (PD 173074), MAPK (U0126, MEK1/2 inhibitor), PI3K (LY294002), PLC $\gamma$ (U73122), p38MAPK (SB203850), JNK (SP600125), NFkB (SN50) were used. Figure legends have an "inh" for inhibitors. (F) Western blots showing the effect of kinase inhibition on BDNFinduced expression of p11 protein ( 5100 a10 gene) in cortical mixed cultures. GAPDH was used as a loading control. Also shown in $\mathbf{F}$ is a schematic diagram summarizing the activation of FGF2 and BDNF-inducible kinase cascades that lead to S100a10 regulation. (G) qRT-PCR analysis of Bdnf, Fgf2 and S100a10 mRNA expression at $12 \mathrm{~h}$ after acute treatment with BDNF (purple bar) and Flx (green bar) in cortical mixed cultures. Statistical analysis in C, D, E, G was done using one-way ANOVA and corrections for multiple comparisons were performed using post-hoc Bonferroni test. Comparisons were made between growth factor-treated samples with and without inhibitors in $\mathbf{C}, \mathbf{D}$ and $\mathbf{E} ; \mathrm{n}=6$. Fold change values for the control vehicle samples are normalized to 1 . Comparisons were made between vehicle- and Flx-treated samples in $\mathbf{G} ; \mathrm{n}=3$. In $\mathbf{D}$ and $\mathbf{E}$, + symbol indicates comparison between vehicle- and BDNF- or FGF-treated, and * symbol indicates comparison between growth factor-treated samples with and without inhibitors. Data are mean $+/-$ SEM; ${ }^{*} P \leq 0.05,{ }^{* *} P \leq 0.01,{ }^{* *} P \leq 0.005$. Dashed lines in $\mathbf{C}$ indicate fold change of the control sample and dashed lines in $\mathbf{D}$ and $\mathbf{E}$ indicate fold change of the FGF2- and BDNF-treated sample. Experiments in $\mathbf{C}, \mathbf{D}, \mathbf{E}, \mathbf{G}$ were reproduced three times with identical results. 
bioRxiv preprint doi: https://doi.org/10.1101/2021.07.23.453443; this version posted July 25, 2021. The copyright holder for this preprint (which

A

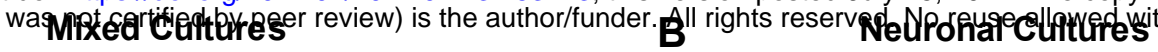
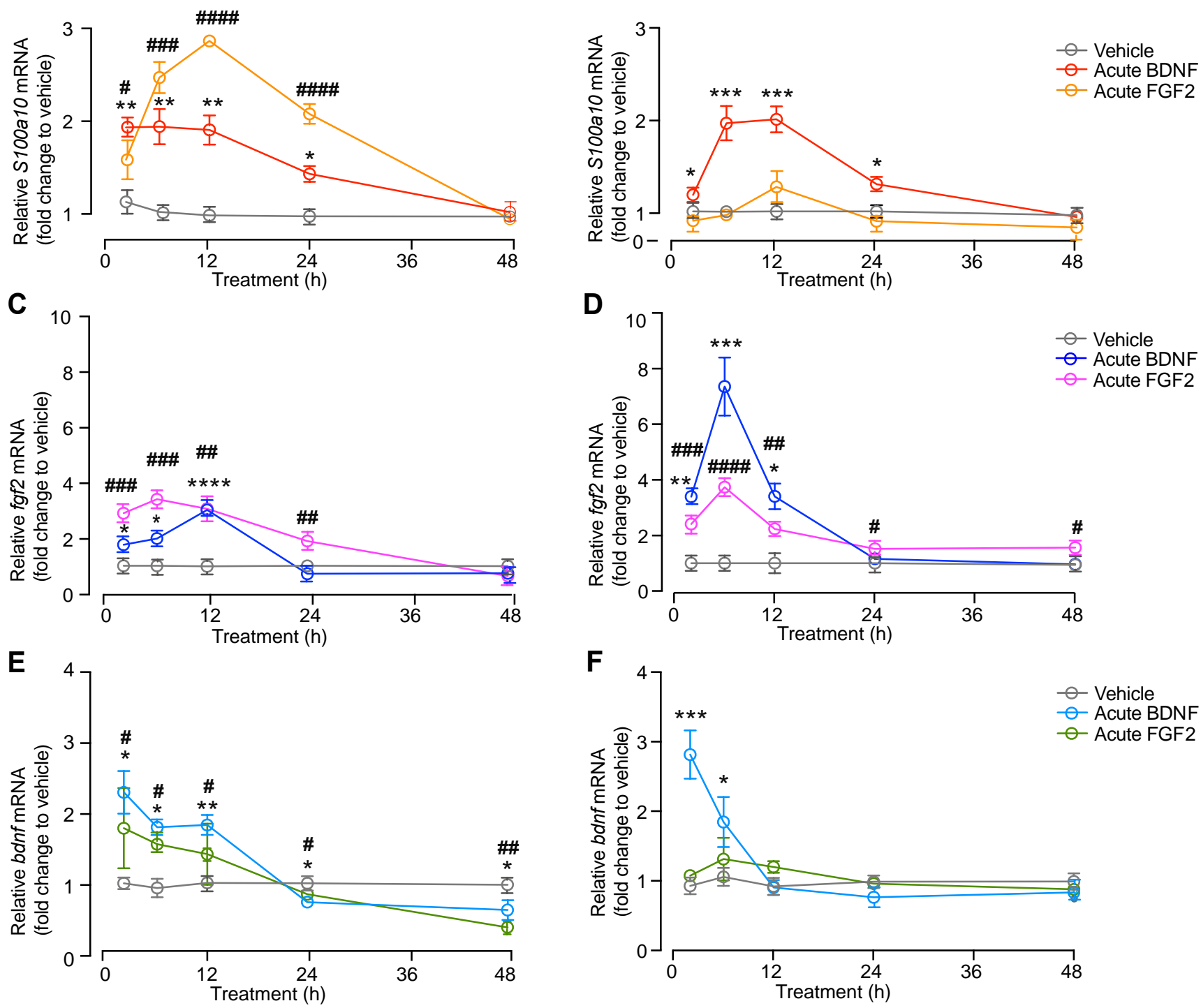

Extended Figure 6. (A) Kinetics of Fgf2, Bdnf and S100a10 mRNA expression at 2h, 5h, 12h, 24h, and 48h after acute treatment with FGF2 and BDNF in DIV7 cortical mixed cultures by qRT-PCR. (B) Kinetics of Fgf2, Bdnf and S100a10 mRNA expression at 2h, 5h, 12h, 24h, and 48h after acute treatment with FGF2 and BDNF in DIV7 cortical neuronal cultures by qRT-PCR. Statistical analysis was done using one-way ANOVA and corrections for multiple comparisons were performed using post-hoc Bonferroni test. Comparisons were made between vehicle- and FGF2 or BDNF-treated samples; $\mathrm{n}=6$. \# symbol indicates comparison between vehicle and FGF2-treated samples, and * symbol indicates comparison between vehicle and BDNF-treated samples. Data are mean $+/$ - SEM; ${ }^{*} P \leq 0.05,{ }^{* *} P \leq 0.01,{ }^{* * *} P \leq 0.005,{ }^{* * * *} P \leq 0.0005$. Experiments were independently reproduced three times with identical results. 